\title{
Typical 2-Cys Peroxiredoxins as a Defense Mechanism against Metal-Induced Oxidative Stress in the Solitary Ascidian Ciona robusta
}

\author{
Laura Drago 1, Diana Ferro 2, Rigers Bakiu ${ }^{3}$, Loriano Ballarin 1,* and Gianfranco Santovito 1,* \\ 1 Department of Biology, University of Padova, 35131 Padova, Italy; laura.drago@phd.unipd.it \\ 2 Department of Pediatrics, University of Missouri-Kansas City, Kansas City, MO 64108; dferro@cmh.edu \\ 3 Department of Aquaculture and Fisheries, Agricultural University of Tirana, 1000 Tiranë, Albania; rigers.ba- \\ kiu@ubt.edu.al \\ * Correspondence: loriano.ballarin@unipd.it (L.B.); gianfranco.santovito@unipd.it (G.S.)
}

\begin{abstract}
Typical 2-Cys peroxiredoxins (2-Cys Prdxs) are proteins with antioxidant properties belonging to the thioredoxin peroxidase family. With their peroxidase activity, they contribute to the homeostatic control of reactive oxygen species (ROS) and, therefore, participate in various physiological functions such as cell proliferation, differentiation, and apoptosis. Although Prdxs have been shown to be potential biomarkers for monitoring the aquatic environments, minimal scientific attention has been devoted to describing their molecular architecture and function in marine invertebrates. Our study aims to clarify the protective role against stress induced by exposure to metals $(\mathrm{Cu}, \mathrm{Zn}$, and Cd) of three Prdxs (Prdx2, Prdx3, and Prdx4) in the solitary ascidian Ciona robusta, an invertebrate chordate. Here we report a detailed pre- and post-translational regulation of the three Prdx isoforms. Data on intestinal mRNA expression, provided by qRT-PCR analyses, show a generalized increase for $\operatorname{Prdx} 2,3$, and 4, which is correlated to metal accumulation. Furthermore, the increase in tissue enzyme activity observed after $\mathrm{Zn}$ exposure is slower than that observed with $\mathrm{Cu}$ and $\mathrm{Cd}$. The obtained results increase our knowledge of the evolution of anti-stress proteins in invertebrates and emphasize the importance of the synthesis of Prdxs as an efficient way to face adverse environmental conditions.
\end{abstract}

Keywords: Ciona robusta; tunicate; metals; typical 2-Cys peroxiredoxins; antioxidant enzymes.

\section{Introduction}

Industrial and mining activities, including the unwise use of pesticides, are considered the primary pollution sources of aquatic bodies. The abiotic factors, including xenobiotics, temperature, and hypo-osmotic stress, have been shown to affect the normal physiology of marine animals and induce physiological responses aimed at maintaining homeostasis [1]. These modifications require the regulation of the expression of stress response genes such as those encoding antioxidant proteins [2-8], and among them peroxiredoxins (Prdxs) $[9,10]$.

Prdxs are thiol-specific antioxidant enzymes that have recently received significant attention for their role in stress responses, characterized by an imbalance between the reactive oxygen species (ROS) production rate and their removal from the cell. These proteins can modulate various physiological functions, such as cell proliferation, differentiation, and apoptosis, by decreasing peroxide levels and the risk of ROS-related damages $[11,12]$. With their peroxidase activity, Prdxs act to reduce and detoxify peroxynitrites (ONOO-), hydrogen peroxide $\left(\mathrm{H}_{2} \mathrm{O}_{2}\right)$, and a wide range of different organic hydroperoxides $(\mathrm{ROOH})$ by using their cysteine residues for catalysis [13-15].

Prdxs are broadly distributed among living organisms, from bacteria to mammals [16-21]. Although they are predominantly located in the cytosol, they have also been found in mitochondria, chloroplasts, and peroxisomes. The first Prdx was discovered in 
1987, in yeast, and named thiol-specific antioxidant (TSA) since it was thought to be able to remove reactive sulfur derivatives (e.g., $\bullet$ RS, $\bullet \mathrm{RSSR}^{-}$, RSOOH) rather than ROS. Subsequently, when TSA was shown to reduce peroxides by using thioredoxin (Trx) as an immediate hydrogen donor, the protein was renamed thioredoxin peroxidase (TPX). Lastly, it was renamed Prdx after realizing that some enzymes of this family do not rely on Trx as electron donors [22].

In mammals, six Prdx isoforms have been identified. They are classified into three subgroups: typical 2-Cys, atypical 2-Cys and 1-Cys [15]. All the Prdxs share the same catalytic mechanism, in which an active-site cysteine (the peroxidatic cysteine, $\mathrm{CP}$ ), containing a sulfur atom (CP-SH), confers the catalytic activity and is oxidized to a sulphenic acid $(\mathrm{CP}-\mathrm{SOH})$ by the peroxide substrate. The recycling of the sulphenic acid back to a thiol is what distinguishes the three enzyme subgroups, in which $C_{P}$ is highly conserved, whereas the "resolving" Cys $\left(C_{R}\right)$ residue can have different positions (2-Cys subgroups) or be absent (1-Cys subgroup) [23,24].

All the Prdx subgroups share a typical core organization containing five $\alpha$-helices and seven $B$-strands [25]. In the reduced form ( $\mathrm{SH})$, the peroxidatic cysteine is in a solventaccessible pocket, formed by a loop-helix structural motif, surrounded by three residues conserved in all the subgroups: $\mathrm{Pro}^{44}{ }^{4} \mathrm{Thr}^{48}$ and $\mathrm{Arg}^{127}$. The atypical 1-Cys Prdx has an additional active site where, besides a histidine $\left(\mathrm{His}^{26}\right)$ and an aspartic acid (Asp ${ }^{140}$ ) residue, a serine $\left(\mathrm{Ser}^{32}\right)$ is present, that allows the phospholipase activity [26]. Prdx1 to 5 belong to the 2-Cys subgroups, containing two conserved cysteines ( $C_{P}$ and $C_{R}$ ). Prdx1 to 4 are included in the typical 2-Cys subgroup. They function as obligate homodimers since they form inter-subunit disulfide bonds between $\mathrm{C}_{\mathrm{P}}-\mathrm{SOH}$ of a subunit and $\mathrm{C}_{\mathrm{R}} \mathrm{SH}$ of another one, then reduced by cell-specific disulfide oxidoreductases to complete the catalytic cycle. Prdx5, included in the atypical 2-Cys subgroup, functions as a monomer, undergoing intra-subunit disulfide bonds, between $\mathrm{C}_{\mathrm{P}}-\mathrm{SOH}$ and $\mathrm{C}_{\mathrm{R}}-\mathrm{SH}$ of the same subunit. The reduction of disulfide bond is operated by Trx [27]. Lastly, Prdx6, included in the 1-Cys subgroup, forms homo- or hetero-dimers. Since it has only one highly conserved cysteine residue, $\mathrm{C}_{\mathrm{p}} \mathrm{SOH}$, it is reduced by thiol-containing electron donor molecules (e.g., glutathione). In general, Prdx levels can be regulated by changes in the phosphorylation, redox and oligomerization states [15].

The measure of intracellular Prdx levels can be used in the biomonitoring of the aquatic environment as a critical indicator of pollution [28]. However, only a few Prdxs have been studied in aquatic invertebrates, mostly in crustaceans and mollusks [29,30], and the modulation of their synthesis by various pollutants needs to be explored. Therefore, this study aims to increase our knowledge on ROS scavenging in invertebrates exposed to high metal ion concentrations, using Ciona robusta as a reference organism.

This marine invertebrate is considered a reliable animal model for evolutionary studies, thanks to its peculiar phylogenetic position of invertebrate chordate closely related to vertebrates [31]. It is also functional to ecotoxicological studies, as it is a filter-feeding organism able to bioaccumulate xenobiotics even at low concentrations [32]. In the tunicate database ANISEED [33], we identified and characterized three transcripts for Prdx2, $\operatorname{Prdx3}$, and Prdx4, respectively. Their genetic expression was analyzed at transcriptional and post-transcriptional level in the intestine of $C$. robusta upon the exposure of animals to copper $(\mathrm{Cu})$, zinc $(\mathrm{Zn})$, and cadmium $(\mathrm{Cd})$ at the same experimental conditions used in previous studies. Finally, we analyzed possible correlation of our results with those previously obtained with other anti-stress proteins, such as metallothioneins [34], the enzymes involved in glutathione (GSH) biosynthesis [35], phytochelatin synthase [36], $\mathrm{Cu} / \mathrm{Zn}$ superoxide dismutase (Cu/Zn SOD) [37], glutathione peroxidase 7 [38] and proteins for stress granules nucleation [39]. 


\section{Materials and Methods}

\subsection{Animals and treatments}

Specimens of C. robusta were sampled in Chioggia, near the Marine Station of the University of Padova, and transferred to the Department of Biology, University of Padova, where they were maintained in aerated aquaria, filled with filtered $(0.45-\mu \mathrm{m})$ seawater (FSW). They were fed with Phyto Marine (Oceanlife, Bologna, Italy) and Reefpearls (D. van Houten, Groningen, The Netherlands) and acclimated for five days at $17^{\circ} \mathrm{C}$, alternating periods of light and darkness $(12 \mathrm{~h} / 12 \mathrm{~h})$. Twenty-four animals were considered for each metal exposure and reared in separate aquaria. Storage solutions of $\mathrm{CuCl}_{2}, \mathrm{ZnCl}_{2}$, and $\mathrm{CdCl}_{2}$ were previously prepared in distilled water and their concentrations were determined with a Perkin Elmer 4000 Atomic Absorption Spectrophotometer (Perkin Elmer, Waltham, MA, USA). The solutions were used to prepare working dilutions with a final sub-lethal concentration of $10 \mu \mathrm{M}$ in FSW. One aquarium filled only with FSW hosted twenty-four animals used as reference controls. After 6, 24, 48, and $72 \mathrm{~h}$ of exposure, six specimens were collected from each aquarium, dissected, and their intestines were frozen in liquid nitrogen and stored at $-80^{\circ} \mathrm{C}$ until use.

\subsection{Primers design, total RNA extraction, cDNA synthesis, amplification, and sequencing}

In the ANISEED database (https://www.aniseed.cnrs.fr/), gene sequences related to C. robusta Prdxs are present. Their identities were validated by BLAST comparison (https://blast.ncbi.nlm.nih.gov/Blast.cgi) to identify those of Prdx2, Prdx3, and Prdx4. Primers for PCR amplification (Table S1) were designed in the coding region of putative Prdx2 (transcript ID: KY2019:KY.Chr8.1384.v1.nonSL16-1), Prdx3 (transcript ID: KY2019:KY.Chr10.1364.v1.ND1-1), Prdx4 (transcript ID: KY2019:KY.Chr10.1036.v1.SL1-5) and $\beta$-actin (transcript ID: KY2019:KY.Chr11.686.v1.nonSL30-1), the latter used as endogenous control. They were analyzed with the IDT Oligo Analyzer tool (https://eu.idtdna.com/pages/tools/oligoanalyzer) and synthesized by BMR genomics SRL (Padova, Italy).

Total RNA was extracted from the intestines of C. robusta by precipitation, using Biozol (Hangzhou Bioer Technology, Hangzhou, China) as lysis buffer according to product manual specifications. RNA concentration and purity were assessed by the A260/280 and A260/230 ratios, with a Nanodrop ND-1000 spectrophotometer (ThermoFisher Scientific, Waltham, MA, USA). Its integrity was proved by visualization of rRNAs in $1 \%$ agarose gel, using Midori Green Direct (Nippon Genetics, Tokyo, Japan) as an intercalating agent and loading dye.

The reverse transcription was performed on $1 \mu \mathrm{g}$ of total RNA, using the ImPromII ${ }^{\mathrm{TM}}$ Reverse Transcription System (Promega, Madison, WI, USA) kit (Oligo-dT Anchor primer is reported in Table S1), as previously described [38]. PCR amplifications were carried out with GRS Taq DNA polymerase ( $5 \mathrm{U} / \mu \mathrm{L}$; Grisp, Porto, Portugal), according to the following cycling parameters: $95^{\circ} \mathrm{C}$ for $2 \mathrm{~min}$, then 40 cycles at $95^{\circ} \mathrm{C}$ for $30 \mathrm{~s}$, melting temperature (Tm) (Table S1) for $30 \mathrm{~s}, 72^{\circ} \mathrm{C}$ for $1 \mathrm{~min}$, and the last step at $72^{\circ} \mathrm{C}$ for $10 \mathrm{~min}$. PCR amplicons were visualized on $1.5 \%$ agarose gel with Midori Green Direct (Nippon Genetics) and purified with the Wizard ${ }^{\circledR}$ SV Gel and PCR Clean-Up System (Promega) kit. The transcription in the intestine of C. robusta for Prdx2, Prdx3, and Prdx4, was confirmed by Sanger sequencing of the PCR amplicons, performed by BMR genomics, on the Applied Biosystems 3730XL 96-capillary DNA Analyzer (Applied Biosystems, Foster City, CA, USA). The sequencing results allowed us to design specific primers for quantitative real-time PCR (qRT-PCR) (Table S2). 


\subsection{Quantitative real-time PCR ( $q R T-P C R$ )}

The transcription levels of Prdx isoform 2, 3, and 4, in metal-exposed animals and control specimens were analyzed by qRT-PCR, using the qPCRBIO SyGreen Mix Separate-ROX kit (PCR Biosystems, Wayne, PA, USA). For each treatment, samples of cDNA from six different animals were considered as biological replicates, and each sample was run three times (technical triplicate). The qRT-PCR primers for the three Prdx isoforms and the housekeeping gene $(\beta$-actin) are reported in Table S2. After a preliminary validation of the primer amplification efficiency (absolute quantification), qRT-PCR was performed on $20 \mu \mathrm{L}$ of SYBR Green-ROX mix, containing $50 \mathrm{ng}$ of cDNA, using the Applied Biosystems 7900-HT Fast Real-Time PCR System with the following cycling parameters: $95^{\circ} \mathrm{C}$ for $2 \mathrm{~min}$ (denaturation), then 45 cycles at $95^{\circ} \mathrm{C}$ for $20 \mathrm{~s}$ and $60^{\circ} \mathrm{C}$ for $1 \mathrm{~min}$ (annealing), and the last phase at $95^{\circ} \mathrm{C}$ for $15 \mathrm{~s}, 60^{\circ} \mathrm{C}$ for $1 \mathrm{~min}, 95^{\circ} \mathrm{C}$ for $15 \mathrm{~s}$ and $60^{\circ} \mathrm{C}$ for $15 \mathrm{~s}$ (extension). The dissociation curve confirmed the absence of genomic contamination. Relative quantification values were obtained using the Pfaffl mathematical model $\left(2^{-\Delta \Delta \mathrm{Ct}} \mathrm{cal}-\right.$ culation) [40]. The amounts of expression level were normalized with respect to the housekeeping gene as in previous studies [37, 38].

\subsection{Tissue activity of 2-Cys Prdxs}

To assess the Prdx2, Prdx3, and Prdx4 activities, intestines from treated and nontreated specimens were homogenized according to 2-Cys Peroxiredoxin Activity Assay protocol (Redoxica, Little Rock, AR, USA). In addition, cell extracts were used to evaluate protein concentration, enzymatic activity, and metal content.

Protein concentration in cell extracts was measured with the Lowry's method, based on the colorimetric reaction with the Folin-Ciocalteu reagent, sensitive to protein quantities of 5-100 $\mu \mathrm{g}$ [41]. Protein quantification was performed with Perkin-Elmer Lambda EZ$201 \mathrm{UV} /$ VIS spectrophotometer at $750 \mathrm{~nm}$.

To determine the 2-Cys Prdx activity in cell extracts, two reaction solutions were prepared, with and without Trx, to determine the disappearance of NADPH caused by the reaction catalyzed by thioredoxin reductase. The decrease of NADPH concentration was measured at $340 \mathrm{~nm}$ with Agilent $8453 \mathrm{UV}$-visible spectrophotometer (Agilent, Santa Clara, CA, USA) for $120 \mathrm{~s}$ after adding $\mathrm{H}_{2} \mathrm{O}_{2}$ as substrate. 2-Cys Prdx activity was evaluated as $\mu \mathrm{mol}$ of oxidated NADPH per min at $25^{\circ} \mathrm{C}$. Data were then normalized against total protein concentration, and bovine serum albumin was used as standard.

\subsection{Analyses of tissue metal content}

$\mathrm{Cu}, \mathrm{Zn}$ and $\mathrm{Cd}$ content in the cell extracts was determined by atomic absorption spectrophotometry. The instrument for metal analyses (Perkin-Elmer mod. 4000) was calibrated by the standard addition method and new standard salt solutions. The concentration obtained was normalized with data of total protein concentration in the same samples.

\subsection{Statistical analyses}

Data are expressed as the mean of six different biological samples $(n=6) \pm$ standard deviation. Statistical analyses were performed with the PRIMER statistical program, using the ANOVA test followed by Duncan's test, to evaluate significant differences between treated and untreated samples $(\mathrm{p}<0.05)$.

\subsection{Gene and protein organization analyses}

Prdx nucleotide sequences for the isoforms 2, 3 and 4 of C. robusta, obtained by amplicon sequencing, were completed in silico, thanks to the ANISEED database, and then translated with the ExPASy translate tool (https://web.expasy.org/translate/). Exon/intron composition was analyzed by matching the cDNA with the genomic sequence found in the ANISEED database, using the Splign tool (https://www.ncbi.nlm.nih.gov/sutils/splign/splign.cgi). Two thousand nt upstream the 
transcript sequence were analyzed with the Primer Premier 5.00 software package (Primer Biosoft International, Palo Alto, CA, USA) to identify putative transcription factor binding sites, such as antioxidant response element (ARE), xenobiotic response element (XRE) and metal response element (MRE). The 3'-UTR regions were analyzed in silico to find putative sequences of rapid degradation and polyadenylation.

The domain composition of Prdxs was predicted with the SMART program (http://smart.embl-heidelberg.de/smart/set_mode.cgi?NORMAL=1). On the alignment, obtained with Clustal Omega (https://www.ebi.ac.uk/Tools/msa/clustalo/), comparing the amino acid sequences of C. robusta and orthologous sequences of other metazoans collected from GenBank (https:/www.ncbi.nlm.nih.gov/nucleotide/), the domain architecture was visualized. The LALIGN tool (https://www.ebi.ac.uk/Tools/psa/lalign/) was used to compare the amino acid sequences of Prdxs two by two, and the NCBI CD-search tool (https://www.ncbi.nlm.nih.gov/Structure/cdd/wrpsb.cgi) was used to classify them correctly.

\subsection{Sequence alignments and phylogenetic analyses}

The cDNA and amino acid sequences of $\operatorname{Prdx} 2, \operatorname{Prd} 33$, and $\operatorname{Prdx} 4$ of C. robusta were used for multiple alignment analyses with the T-Coffee package (Comparative Bioinformatics Group, Barcelona, Spain) [42], together with orthologous sequences of other metazoans, available in GenBank and ANISEED databases (Tables S3). T-Coffee was used to align Prdx2, Prdx3 and Prdx4 sequences. Even though the method is based on the popular progressive approach to multiple alignments, it is characterized by a dramatic improvement in accuracy with a modest sacrifice in speed compared to the most used alternatives.

jModelTest 2.0 [43] was used to select the best-fit model of nucleotide substitution systematically. Analyses were performed using 88 candidate models and three types of criteria (Akaike information criterion (AIC), corrected Akaike information criterion (cAIC), and Bayesian information criterion (BIC)). Finally, ProtTest 3 [44] was used to select the best-fit model to analyze protein evolution. One hundred and twenty-two candidate models and the three previously mentioned criteria were used in these statistical analyses.

Phylogenetic trees were built using the Bayesian inference (BI) method implemented in Mr. Bayes 3.2 [45]. Four independent runs, each with four simultaneous Markov Chain Monte Carlo (MCMC) chains, were performed for 1.000.000 generations sampled every 1000 generations. Furthermore, we also used the maximum likelihood (ML) method implemented in PhyML 3.0 [46]. Bootstrap analyses were performed on 100.000 trees using nearest neighbor interchange (NNI) as a method for improvement of tree topology. FigTree v1.4.4 software (GitHub, San Francisco, CA, USA; http://ree.bio.ed.ac.uk/software/figtree/) was used to display the annotated phylogenetic trees.

\subsection{In situ hybridization (ISH)}

Amplicons obtained with primers designed for the qRT-PCR (Table S2) were ligated in pGEM T-Easy Vector (Promega) and cloned in XL1-Blue Escherichia coli cells (Invitrogen, Thermo Fisher Scientific, Waltham, MA, USA). UltraPrep (AHN Biotechnologie, Nordhausen, Germany) kit was used to extract plasmid DNA from positively screened colonies, which was then sequenced by Eurofins Genomics (Eurofins Genomics Ebersberg, Germany) to check the successful ligation of the insert. Sense and anti-sense riboprobes were synthesized with the SP6 Polymerase (Promega) kit and purified with miniQuick Spin Columns ${ }^{\mathrm{TM}}$ (Roche Molecular Systems, F. Hoffmann-La Roche AG, Basel, Switzerland).

As carried out in previous studies, ISH was performed on hemolymph extracted from animals exposed for 3 days to metal (FSW in controls) [39]. After pelleting the cells by centrifuging ( $800 \times \mathrm{g}$ for $10 \mathrm{~min}$ ), hemocytes were resuspended in FSW to a concentration of $10^{5}$ cells/mL and left to adhere on Superfrost Plus glass slides (Menzel-Glaser, Thermo Fisher Scientific, Waltham, MA, USA) for $30 \mathrm{~min}$. After fixation with a freshly prepared 
solution of $4 \%$ paraformaldehyde, $0.1 \%$ glutaraldehyde in $0.2 \mathrm{M}$ cacodylate buffer, $\mathrm{pH} 7.4$, for $30 \mathrm{~min}$, cells were washed in phosphate-buffered saline (PBS: $1.37 \mathrm{M} \mathrm{NaCl}, 0.03 \mathrm{M} \mathrm{KCl}$, $0.015 \mathrm{M}$ KH2PO4, $0.065 \mathrm{M} \mathrm{Na} 2 \mathrm{HPO} 4, \mathrm{pH} 7.2)$, permeabilized in $0.1 \%$ Triton- $X$ in PBS for 5 min and washed again in PBS. The prehybridization step was performed with the Hybridization Cocktail 50\% Formamide (Amresco, VWR International, Radnor, PA, USA) for $1 \mathrm{~h}$ at $58^{\circ} \mathrm{C}$, followed by hybridization, at the same temperature, in the same solution with $2 \mu \mathrm{g} / \mathrm{ml}$ of biotin-labeled riboprobe overnight. Next, cells were washed in SSC (0.3 M $\mathrm{NaCl}, 40 \mathrm{mM}$ sodium citrate, $\mathrm{pH} 4.5$ ), then three times in 50\% formamide in SSC for 30 min each at $58^{\circ} \mathrm{C}$ and finally in PBS containing $0.1 \%$ Tween-20 (PBS-T). Samples were incubated in the dark in a solution of $5 \% \mathrm{H}_{2} \mathrm{O}_{2}$ in methanol for $30 \mathrm{~min}$, washed with PBS-T, and treated with Vectastain ABC (Vector Laboratories, Burlingame, CA, USA) for 30 min. Cells were stained with 0.025\% 3,3'-diaminobenzidine (DAB; Sigma-Aldrich, Merck, Darmstadt, Germany) and $0.004 \% \mathrm{H}_{2} \mathrm{O}_{2}$ in PBS for 15 min in the dark, dehydrated with ethanol, and mounted with Eukitt (Electron Microscopy Sciences, Hatfield, PA, USA).

\section{Results}

\subsection{Gene and transcript organizations}

The sequencing of the amplicon obtained with CrPrdx $2 \mathrm{fw}-\mathrm{CrPrdx} 2 \mathrm{rv}$ primers on a cDNA from $C$. robusta intestine returned a partial sequence of $478 \mathrm{nt}$, belonging to the putative Prdx2 nucleotide sequence of C. robusta, previously collected from the ANISEED database and used to design the primers reported above. This result suggests that the gene, named $c r-\operatorname{Prdx} 2$ and present in chromosome 8, is transcribed in C. robusta. The in silico analysis indicated that the whole transcript is $667 \mathrm{nt}$ in length, with $5^{\prime}$ - and $3^{\prime}$-UTR regions of $9 \mathrm{nt}$ and $64 \mathrm{nt}$ respectively. The open reading frame (ORF) is $594 \mathrm{nt}$ long and encodes a putative protein of $197 \mathrm{aa}$, with a deduced molecular weight of $21.81 \mathrm{kDa}$. The gene is organized in four exons and three introns, with the ATG start codon located in the first exon, $114 \mathrm{nt}$ long, and the TAA stop codon located in the last one, $278 \mathrm{nt}$ long. In the 3'-UTR region, two nucleotide sequences, corresponding to rapid degradation (ATTTA) signals, are conserved at nt 635 and 641 (Figure S1). In the promoter region of cr- $\operatorname{Prdx2}$, no regulatory sequences ascribable to ARE or MRE were found.

Regarding Prdx3, the amplicon obtained with CrPrdx3fw-CrPrdx3rv primers allowed us to identify a partial sequence of $631 \mathrm{nt}$ included in the sequence found in ANISEED thus confirming that the gene, named $c r-P r d x 3$ and located in chromosome 10, is transcribed in the intestine of C. robusta. The in silico analysis indicated that the whole transcript is $1190 \mathrm{nt}$ in length and contains an ORF of $708 \mathrm{nt}$, flanked by 5'- and 3'-UTR regions of $120 \mathrm{nt}$ and $362 \mathrm{nt}$, respectively. The ORF encodes a putative protein of 235 aa, with a deduced molecular weight of $26.10 \mathrm{kDa}$. The gene is organized in five exons, with the ATG start codon located in the first exon, $162 \mathrm{nt}$ long, and the TAA stop codon located in the last one, $413 \mathrm{nt}$ long. In the 3'-UTR, three conserved sequences correspond to polyadenylation (AATAAA) signals (nt 914, 967, and 1164) and six to rapid degradation (ATTTA) signals (nt 864, 892, 945, 976, 994, and 1001) are present (Figure S2). Furthermore, in the promoter region of $c r-P r d x 3$, one putative ARE (conserved core TGACNNNGC), $1601 \mathrm{nt}$ upstream the start codon, and one putative MRE (conserved core TGCRCNC), 451 nt upstream the start codon, were identified.

Sequencing of the amplicon obtained with $\mathrm{CrPrdx} 4 \mathrm{fw}-\mathrm{CrPrdx} 4 \mathrm{rv}$ primers returned a partial sequence of $585 \mathrm{nt}$. The putative Prdx4 sequence from the ANISEED database contains the sequence we obtained with the amplicon sequencing, thus confirming that the transcription of the gene, named $c r-P r d x 4$ and localized in chromosome 10, occurs in C. robusta intestine. The whole transcript is $1241 \mathrm{nt}$ long, with an ORF of $720 \mathrm{nt}$, located between a 5'-UTR region of $60 \mathrm{nt}$ in length and a $3^{\prime}$-UTR region of $461 \mathrm{nt}$ in length. The putative protein contains 239 aa and has a deduced molecular weight of $26.83 \mathrm{kDa}$. The gene is organized in a single exon of $1240 \mathrm{nt}$. In the $3^{\prime}$-UTR, there is a conserved sequence corresponding to polyadenylation (AATAAA) signal, at nt 1162, and three conserved 
rapid degradations (ATTTA) signals, at nt 972, 994, and 1125 (Figure S3). In addition, putative conserved transcriptional regulation motifs were found in the promoter region of cr-Prdx4: one putative ARE, 1514 nt upstream the start codon, and one putative MRE, 364 nt upstream the start codon.

\subsection{Protein organizations}

The NCBI Conserved Domain Search tool revealed that all the three Cr-Prdx isoforms belong to the thioredoxin proxidase-like superfamily, to the typical 2-Cys Prdx subfamily (Cr-Prdx2 E-value 1.37e-117; Cr-Prdx3 1.36e-117; Cr-Prdx4 2.70e-117). Orthologous amino acid sequences of invertebrates and vertebrates were considered for the multi-alignment analyses, obtained with Clustal Omega, for all the three Prdx isoforms, including C. robusta (Figure S4, S5, and S6; transcript IDs are reported in Table S3). Prdx2, 3 and 4, present two characteristic domains: alkyl hydroperoxide reductase (AhpC)- thiol specific antioxidant (TSA) domain, where the AhpC domain is responsible for directly reducing organic hyperoxides, and the TSA domain constitutes an enzymatic defense against sulfur-containing radicals, and C-terminal 1-cysPrdx domain, crucial for providing the extra cysteine necessary for dimerization of the whole molecule. Loss of the peroxidase activity is associated with oxidation of the catalytic cysteine found upstream of this domain.

Regarding Prdx2, in the AhpC-TSA domain, the 34.3\% of amino acid residues are completely conserved among the examined species. In the C-terminal domain, $31.6 \%$ of the amino acids are completely conserved (Figure S4). In this alignment, the putative CrPrdx2 amino acid sequence shows the highest identity with those of Danio rerio and Phyton bivittatus (74.6\%) and the highest similarity with that of Mus musculus (92.4\%) (Table S4), as revealed by the LALIGN tool.

The AhpC-TSA domain of Prdx3 shows 17.9\% of conserved amino acids, whereas, in the C-terminal domain, they are $36.8 \%$ completely conserved (Figure S5). The putative CrPrdx3 amino acid sequence shows the highest identity (72.4\%) with Xenopus laevis orthologous sequence and the highest similarity (90.1\%) with Drosophila melanogaster orthologous sequence (Table S5).

For Prdx4 (Figure S6), the AhpC-TSA domain results in $60.4 \%$ conservation among the species considered for multi-alignment, whereas the C-terminal domain shows $29.4 \%$ conservation. The putative Cr-Prdx4 amino acid sequence shows the highest identity (88.9\%) and similarity (96.6\%) with Botrylloides leachii orthologous sequence (Table S6).

In all the analyzed peroxiredoxins, the four amino acids responsible for the peroxidase activity were identified: proline (Pro44 in Cr-Prdx2, Pro81 in Cr-Prdx3, and Pro79 in CrPrdx3), threonine (Thr 48 in Cr-Prdx2, Thr 85 in Cr-Prdx3, and Thr83 in Cr-Prdx4), cysteine (Cys51 in Cr-Prdx2, Cys88 in Cr-Prdx3 and Cys86 in Cr-Prdx4) and arginine (Arg 127 in CrPrdx2, Arg164 in Cr-Prdx3 and Arg162 in Cr-Prdx4) (Figure S4, S5 and S6).

\subsection{Molecular phylogeny}

jModelTest 2.0 software indicated that the GTR+I+G model is the best-fit model to analyze the evolution of the coding sequences of all the Prdx isoforms, with a gamma shape value (four rate categories) of 0.868 using all statistical criteria: AIC, cAIC and BIC $(-\ln L=30,440.51)$. Figure 1 shows all the Prdx isoforms phylogenetic tree generated by applying the BI and ML methods to the data set of coding sequences.

The cladogram shows that two significant clusters, corresponding to Prdx3 and Prdx4, are well defined and separated from the Prdx2 sequences (posterior probability $98 \%$, bootstrap value 50\%). However, there are some exceptions, as in Clonorchis sinensis, Aplysia californica and Culex pipiens quinquefasciatus Prdx2s that cluster within the Prdx3 clade and Crassostrea gigas Prdx2, which is included inside the Prdx4 clade. It is to note that Prdx2 of B. leachii emerges with the Prdx4 of this species (posterior probability 100\%, bootstrap value 100\%), and the Prdx4 sequences from other tunicates (Ciona intestinalis and Ciona robusta; posterior probability 100\%, bootstrap value $88 \%$ ). Conversely, within 
the Prdx3 clade, all tunicate sequences clustered together with urinary blood fluke Schistosoma haematobium (posterior probability 78\%). In Prdx2s, species of the genus Ciona grouped in a clade (posterior probability $100 \%$, bootstrap value $100 \%$ ) well separated from the other Prdx2 sequences (posterior probability 100\%).

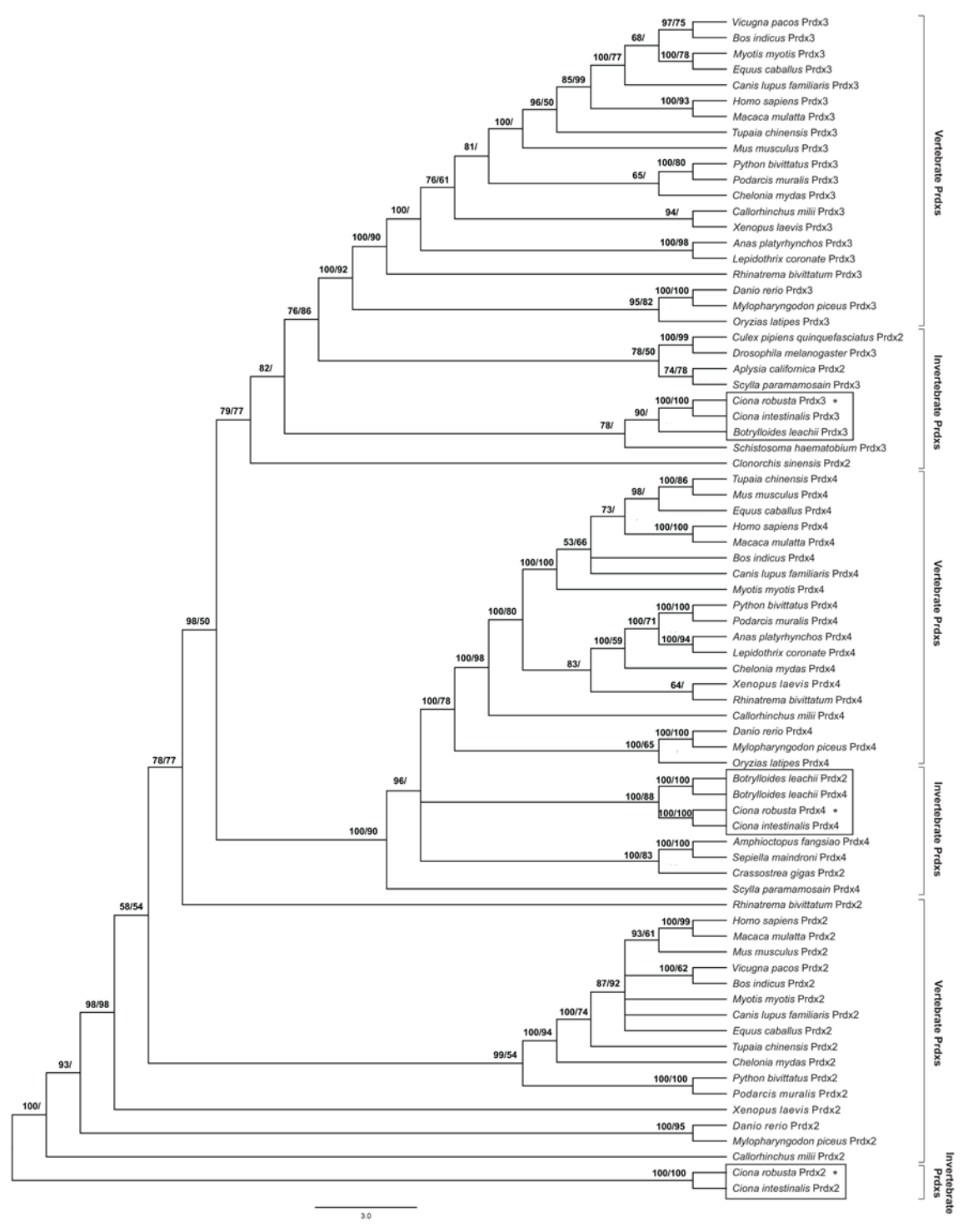

Figure 1. Phylogenetic relationships among Prdxs of various organisms reconstructed on the basis of the cDNA coding region sequences and using both Bayesian interference (BI) and Maximum likelihood (ML) methods. Bayesian posterior probability (first number) and bootstrap values higher (and equal to) than $50 \%$ are indicated on each node, respectively. The scale for branch length (3.0 substitution/site) is shown below the tree.

ProtTest3 statistical results determined that the WAG+G model was the best model to apply for the phylogenetic analysis of Prdx amino acid sequences, with a gamma shape value (four rate categories) of 0.444 using all statistical criteria $(-\ln L=13,169.13)$. Figure S7 shows a Prdx phylogenetic tree generated by applying the BI and ML methods to the data set of amino acid sequences. The cladogram topology is quite similar to the previous one, though in this cladogram three clades are identified, each including the sequences of a specific isoform, with the same exceptions previously highlighted (in this case Clonorchis sinensis Prdx2 emerged close to Prdx4s). 


\section{4. $q R T-P C R$}

The three genes, $c r-P r d x 2, c r-P r d x 3$, and $c r-P r d x 4$, are transcribed in the C. robusta intestine, both in control conditions and after metal exposures. Their gene expression levels are reported in Figures 2, 3, and 4.

The levels of Prdx2 mRNA measured in organisms that were exposed to $\mathrm{Cu}$ showed a time-dependent increase starting from $24 \mathrm{~h}$ (Figure 2a). At $72 \mathrm{~h}$ they were about three times higher compared to the controls $(\mathrm{p}<0.001)$. In the specimens exposed to $\mathrm{Zn}, \operatorname{Prdx} 2$ messenger levels were consistently higher than the controls (Figure 2b). The maximum expression was measured at $6 \mathrm{~h}$, almost three times higher than the controls $(\mathrm{p}<0.01)$. At $24 \mathrm{~h}, 48 \mathrm{~h}$ and $72 \mathrm{~h}$ the expression values decreased slightly, remaining however double compared to the controls $(\mathrm{p}<0.01)$. Exposure to $\mathrm{Cd}$ also determined a statistically significant $(\mathrm{p}<0.01)$ increase in the Prdx2 mRNA levels, compared to the controls, for all the considered times (Figure 2c). The time-course of messenger accumulation was characterized by an initial overexpression followed by a down-regulation at $24 \mathrm{~h}$, and a subsequent new increase at $48 \mathrm{~h}$ when the maximum mRNA expression for Prdx2 was recorded (about ten times compared to the controls). Subsequently there was a further decrease, until the return to the levels measured at $6 \mathrm{~h}$.

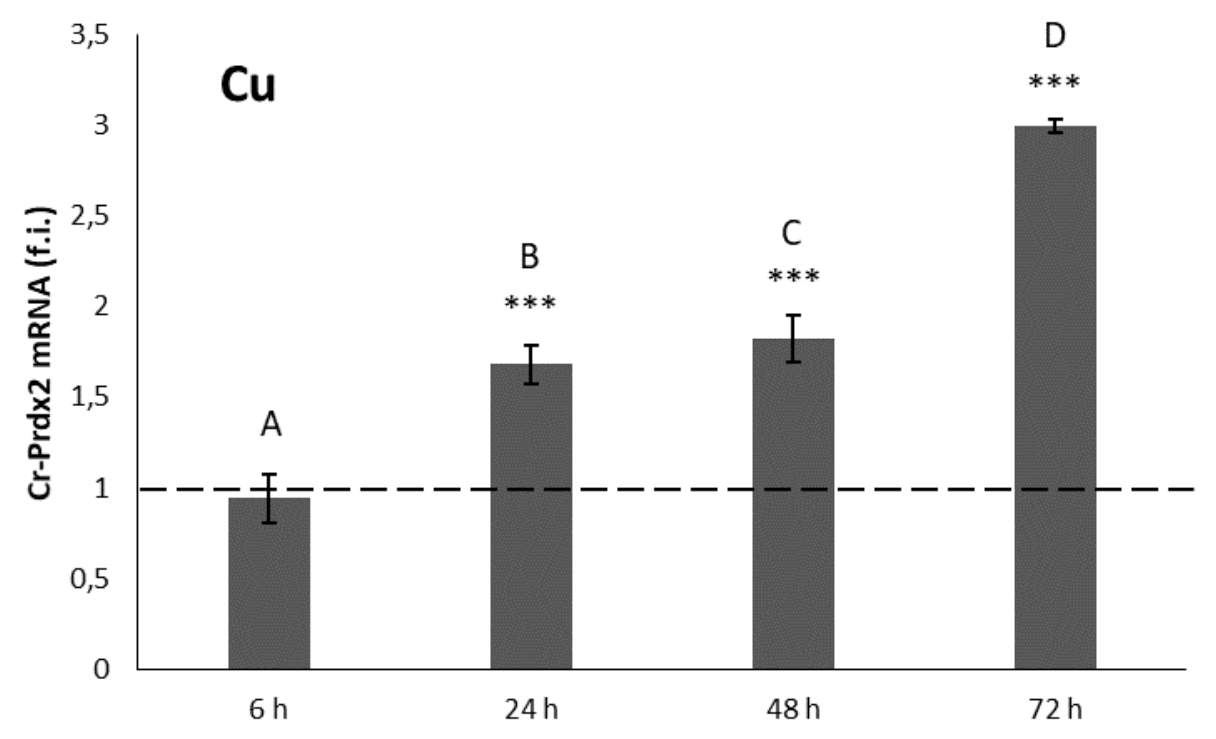

(a)

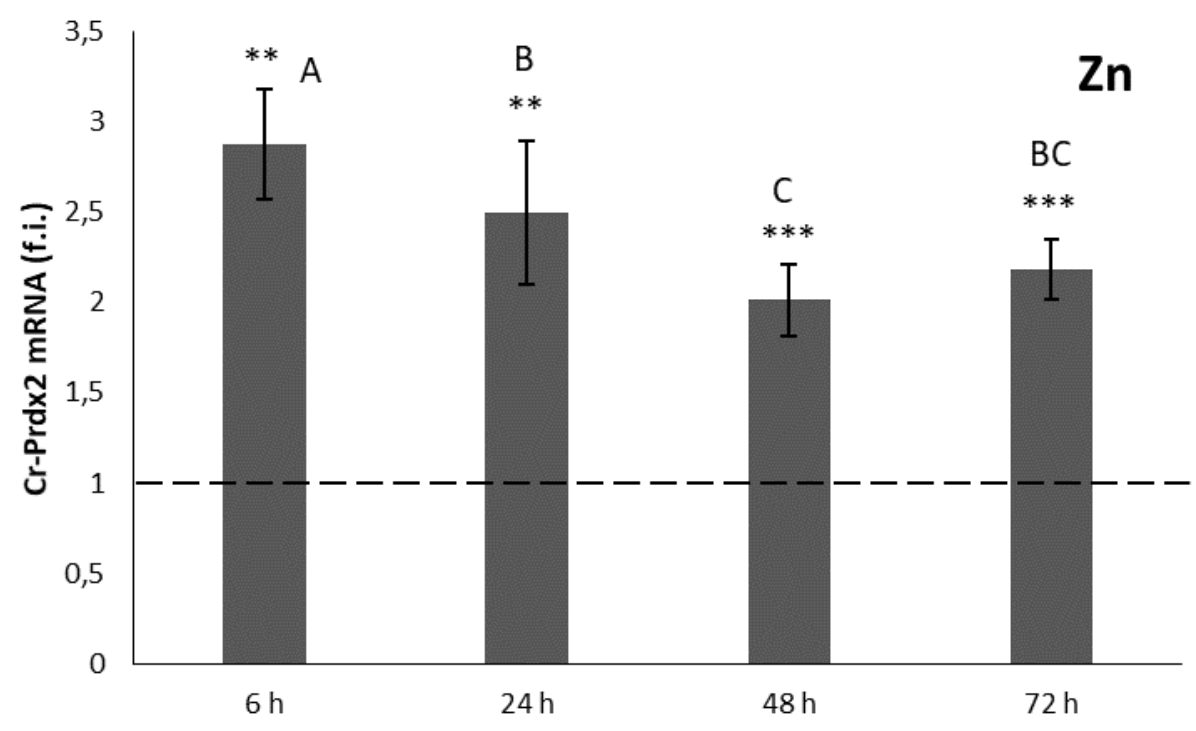


(b)

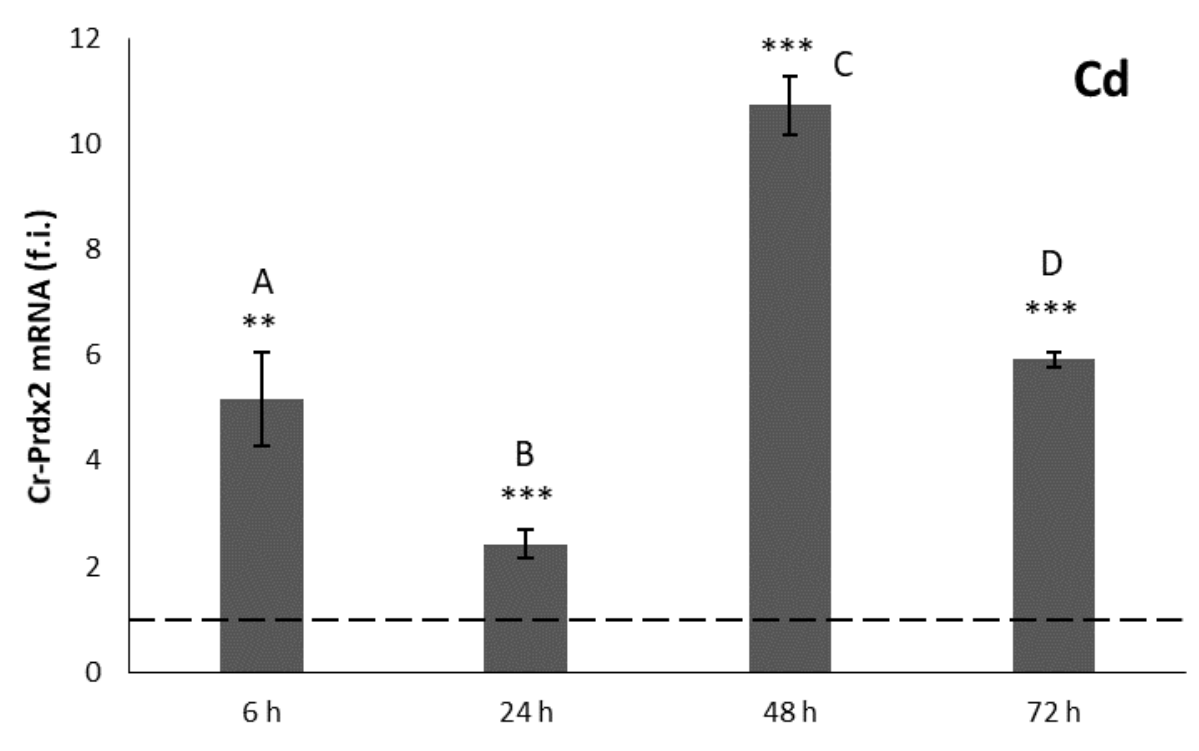

(c)

Figure 2. Relative expression levels (fold induction, f.i.) of $\mathrm{Cr}$-Prdx2, during (a) $\mathrm{Cu}$, (b) $\mathrm{Zn}$, and (c) $\mathrm{Cd}$ exposure. Transcription levels were normalized with respect to controls (dashed line). Asterisks: significant differences with respect to controls $\left.{ }^{* * *} \mathrm{p}<0.001,{ }^{* *} \mathrm{p}<0.01,{ }^{*} \mathrm{p}<0.05\right)$. Letters with the same index: Student-Newman-Keuls t-comparison among treatments.

In the specimens of $C$. robusta exposed to $\mathrm{Cu}$ (Figure 3a), the levels of Prdx3 mRNA remained almost constant throughout the experiment, being approximately $50 \%$ greater than those measured in the controls only at $24 \mathrm{~h}$ and $72 \mathrm{~h}(\mathrm{p}<0.05)$. The mRNA levels for $\operatorname{Prdx} 3$ were consistently higher than the controls in the organisms exposed to $\mathrm{Zn}$ (Figure $3 b)$. Maximum expression was measured at $6 \mathrm{~h}$, with an increase in expression almost three times greater than the respective control $(\mathrm{p}<0.001)$. At $24 \mathrm{~h}, 48 \mathrm{~h}$ and $72 \mathrm{~h}$ the expression values slightly decreased, remaining however higher than the controls. In organisms treated with $\mathrm{Cd}$, there were no statistically significant differences compared to the controls except at $6 \mathrm{~h}(\mathrm{p}<0.01)$, where the level of Prdx3 mRNA was also statistically higher than at the other treatment times (Figure $3 \mathrm{c}$ ).

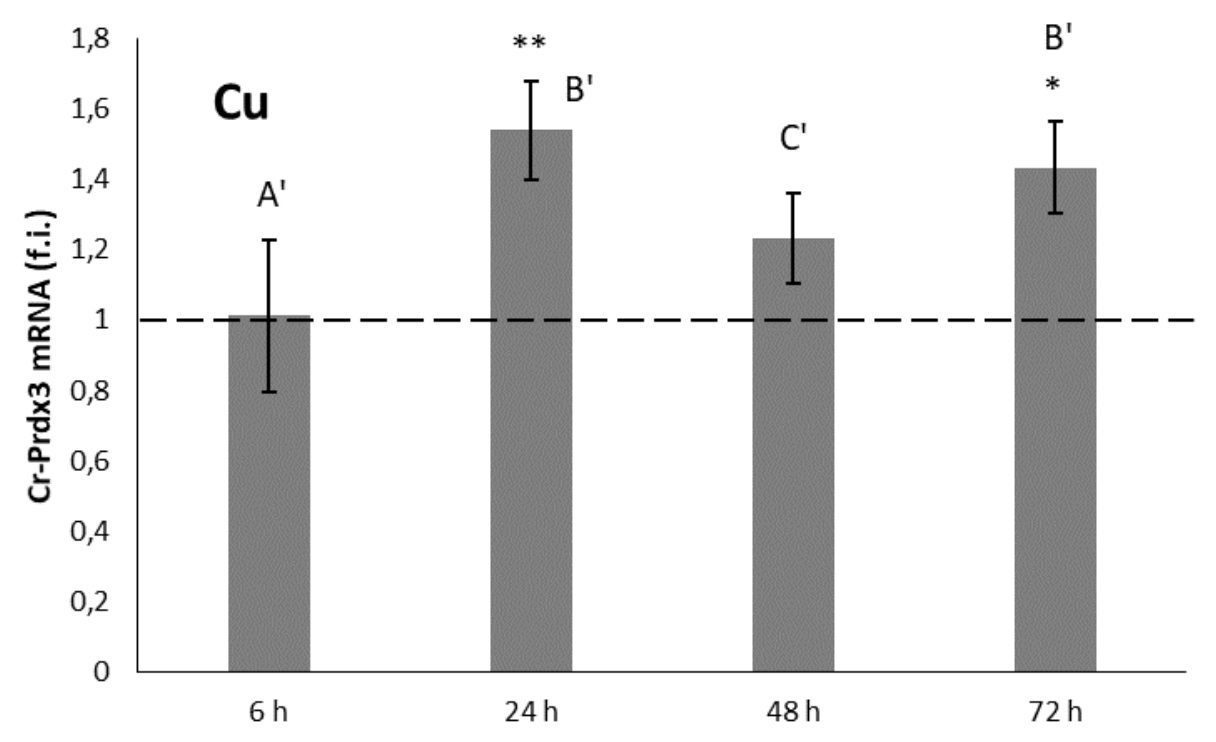

(a) 


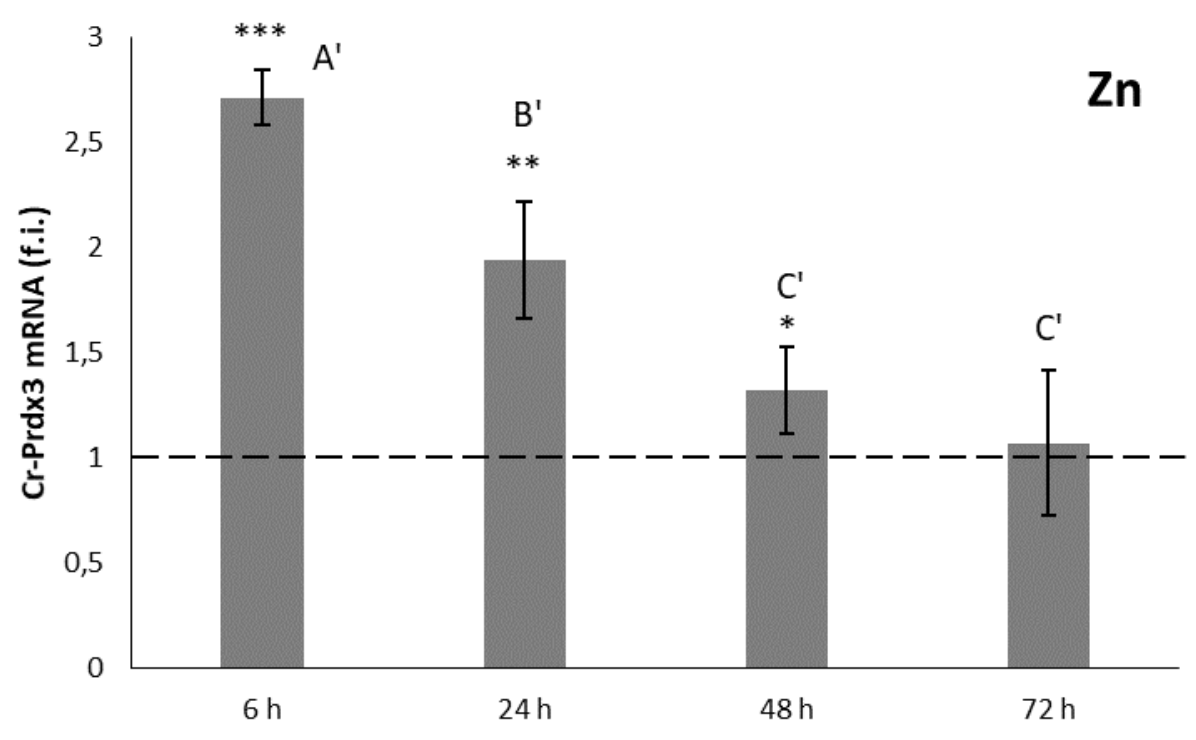

(b)

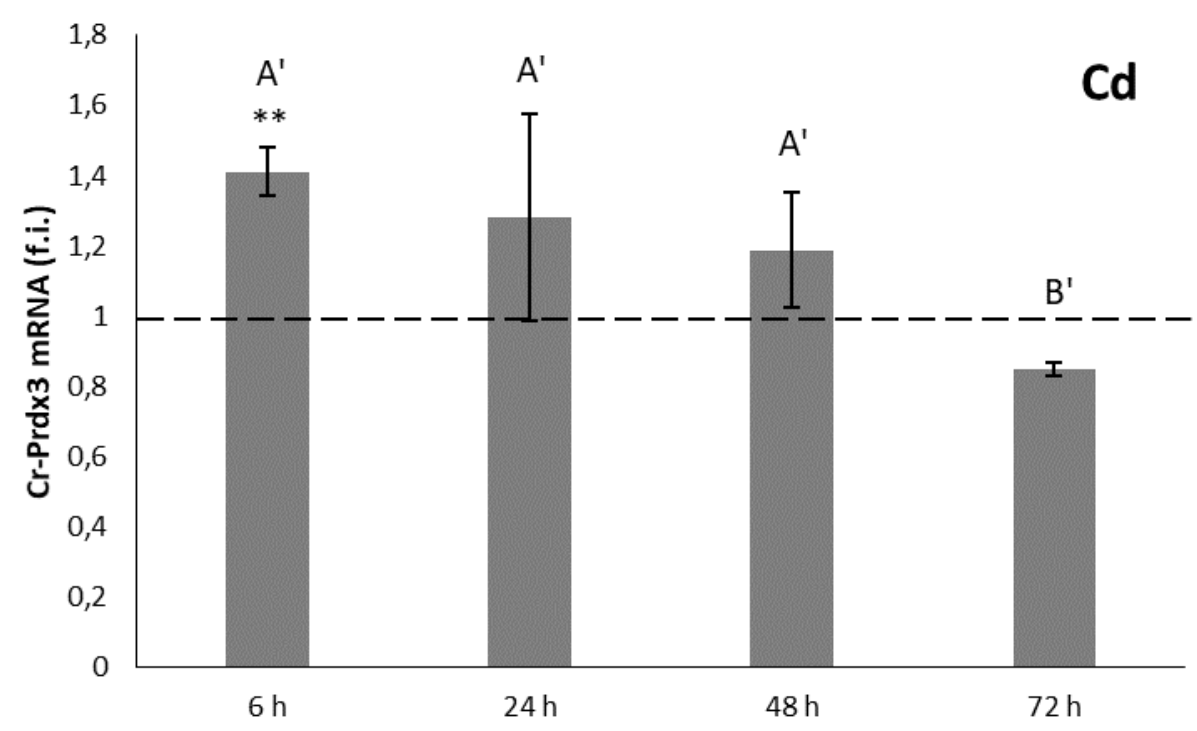

(c)

Figure 3. Relative expression levels (fold induction, f.i.) of $\mathrm{Cr}-\mathrm{Prdx} 3$, during (a) $\mathrm{Cu}$, (b) Zn, and (c) $\mathrm{Cd}$ exposure. Transcription levels were normalized with respect to controls (dashed line). Asterisks: significant differences with respect to controls $\left({ }^{* * *} \mathrm{p}<0.001,{ }^{* *} \mathrm{p}<0.01,{ }^{*} \mathrm{p}<0.05\right)$. Letters with the same index: Student-Newman-Keuls t-comparison among treatments.

Prdx4 mRNA levels measured in Cu-exposed organisms showed a statistically significant increase starting at $24 \mathrm{~h}$ (Figure $4 \mathrm{a}$ ). At $72 \mathrm{~h}$ they were about two times higher than the controls $(\mathrm{p}<0.001)$. In organisms exposed to $\mathrm{Zn}$, the messenger levels for Prdx4 remained consistently higher than the controls $(\mathrm{p}<0.01)$ (Figure $4 \mathrm{~b}$ ). Exposure to $\mathrm{Cd}$ also resulted in a statistically significant $(p<0.01)$ increase compared to the controls of the Prdx4 messenger levels for all the times considered (Figure 4c). The time-course of the accumulation of mRNA in the treated specimens showed an initial overexpression (about three times compared to the controls), which remained constant up to $48 \mathrm{~h}$. Subsequently, there was a down-regulation which resulted in values more similar to those measured in untreated organisms. 


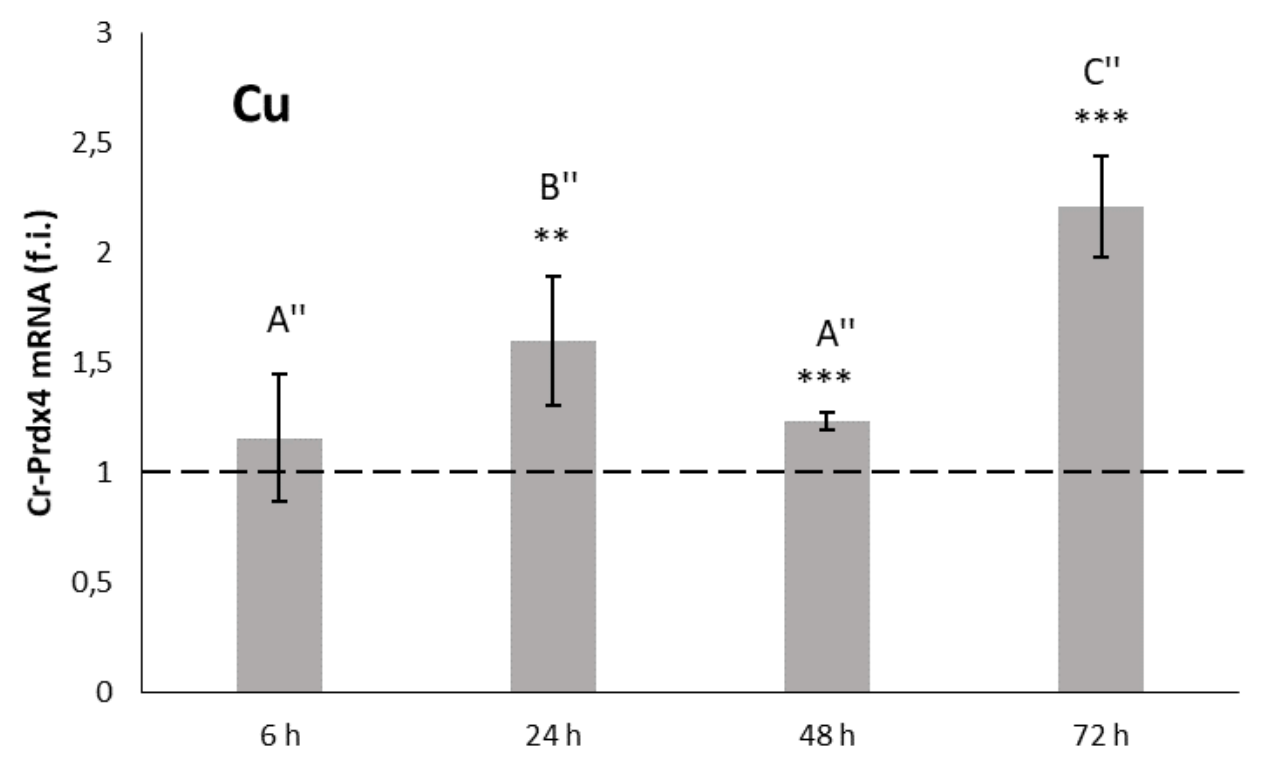

(a)

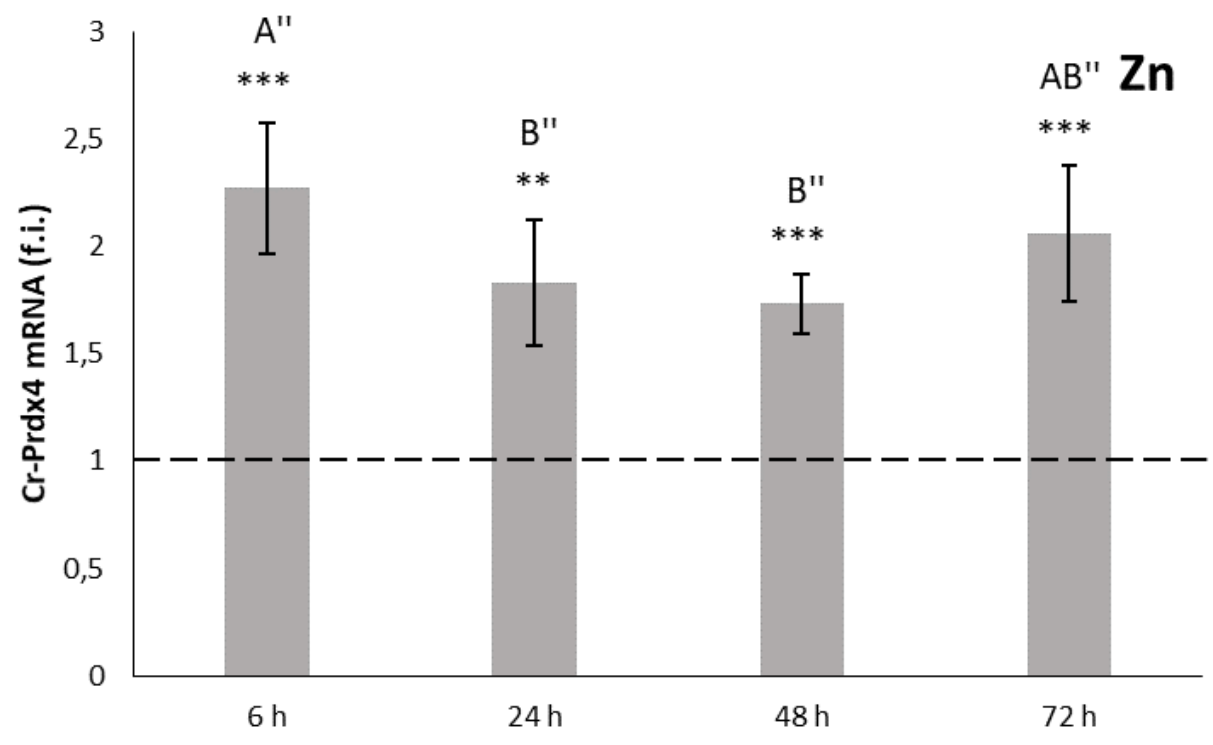

(b) 


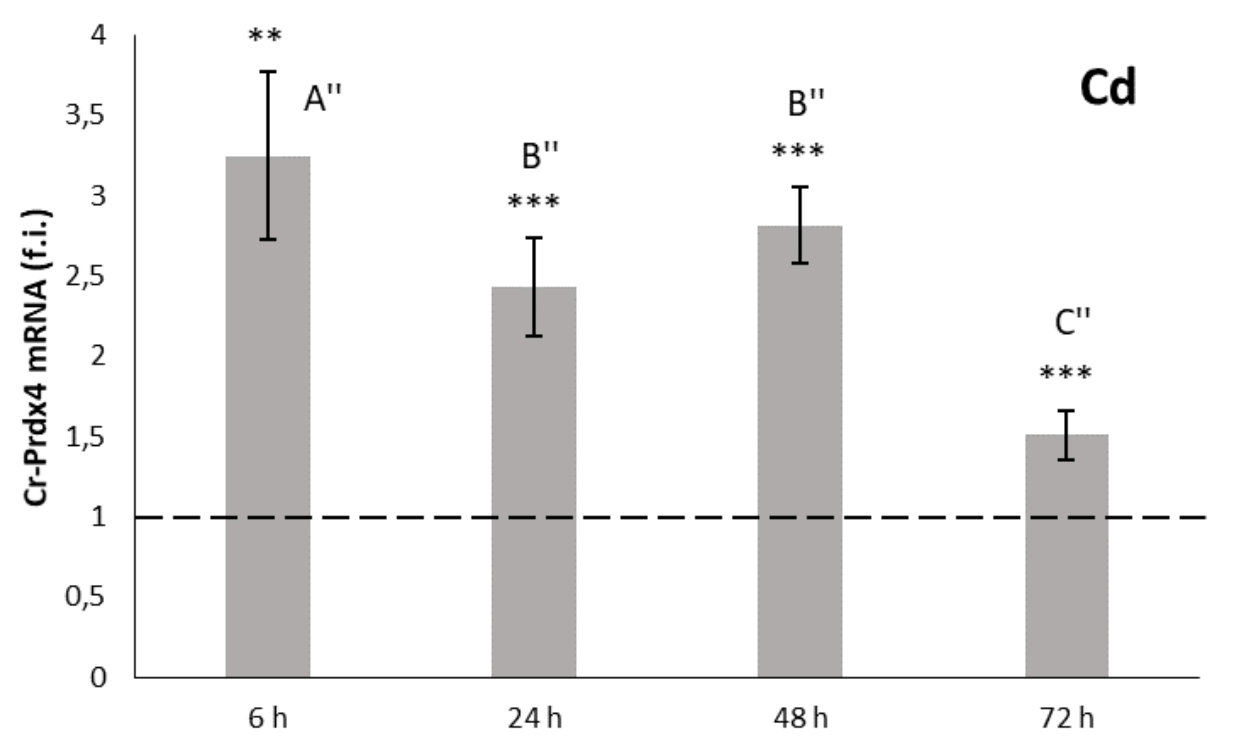

(c)

Figure 4. Relative expression levels (fold induction, f.i.) of Cr-Prdx4, during (a) Cu, (b) Zn, and (c) $\mathrm{Cd}$ exposure. Transcription levels were normalized with respect to controls (dashed line). Asterisks: significant differences with respect to controls $\left({ }^{* *} \mathrm{p}<0.001,{ }^{* *} \mathrm{p}<0.01,{ }^{*} \mathrm{p}<0.05\right)$. Letters with the same index: Student-Newman-Keuls t-comparison among treatments.

\subsection{2-Cys Prdx activity}

We measured the 2-Cys Prdx activity on cell-free extracts of $C$. robusta intestine. Exposure to $\mathrm{Cu}$ led to a statistically significant $(\mathrm{p}<0.001)$ increase in the levels of 2 -Cys Prdx active protein, for all the considered times (Figure 5). In particular, there was a regular increase up to $48 \mathrm{~h}$, which was followed by a marked decrease at $72 \mathrm{~h}$, when a halved tissue activity was found.

In organisms exposed to $\mathrm{Zn}$, the levels of active protein remained unchanged with respect to untreated organisms up to $24 \mathrm{~h}$ (Figure 5). Starting from $48 \mathrm{~h}$, there was a progressive increase in tissue activity, at first three times higher than the controls $(\mathrm{p}<0.001)$ and about five times higher $(\mathrm{p}<0.001)$ at $72 \mathrm{~h}$, when maximum expression was recorded.

For organisms exposed to $\mathrm{Cd}$, statistically significant variations in the levels of 2-Cys $\operatorname{Prdx}$ active protein were found only at $6 \mathrm{~h}$ and $48 \mathrm{~h}$, with values respectively of three and six times higher than in the controls $(p<0.001)$. These peaks of expression were interspersed with downregulation events that brought 2-Cys Prdx activity back to the values measured in untreated specimens. 


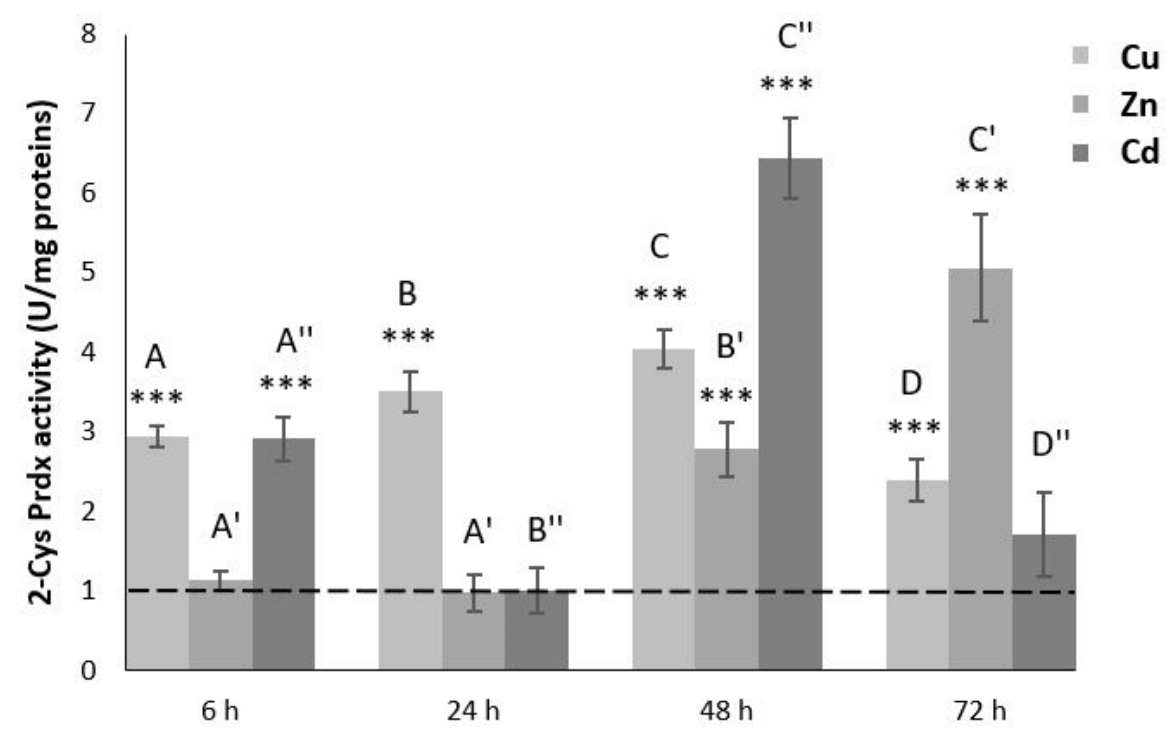

Figure 5. 2-Cys Prdx activity levels in the intestine C. robusta during metal exposure ( $\mathrm{Cu}, \mathrm{Zn}$ and Cd). Protein levels were normalized with respect to controls (dashed line). Asterisks: significant differences with respect to controls ( $\left.{ }^{* * *} p<0.001,{ }^{* *} p<0.01,{ }^{*} p<0.05\right)$. Letters with the same index: Student-Newman-Keuls t-comparison among treatments.

\subsection{Metal contents}

The accumulation of $\mathrm{Cu}$ in organisms exposed to this metal has been shown to be greater than values found in untreated organisms, for all the times considered. At $6 \mathrm{~h}$ and $24 \mathrm{~h}$ the increase in $\mathrm{Cu}$ concentration is about three times $(\mathrm{p}<0.01)$, at $48 \mathrm{~h}$ there is a further five times increase ( $<<0.01)$, and finally at $72 \mathrm{~h}$ the maximum concentration value, well thirty times higher $(\mathrm{p}<0.001)$ than that measured in the controls (Figure 6a).

Instead, in the cell-free extracts of organisms exposed to $\mathrm{Zn}$, significant variations in concentrations of this metal emerged only at $6 \mathrm{~h}$ and $48 \mathrm{~h}$, with values of three and two times higher $(p<0.001)$ than in the controls, respectively (Figure $6 b)$. These peaks of accumulation were interspersed with times when the $\mathrm{Zn}$ concentration was quite similar to that measured in the untreated specimens.

In organisms exposed to $\mathrm{Cd}$ there is a slight increase with respect to controls ( $\mathrm{p}$ $<0.001$ ) in the concentrations of this metal from $24 \mathrm{~h}$ to $72 \mathrm{~h}$ (Figure 6c). The time-course of the $\mathrm{Cd}$ accumulation was characterized by increasing values up to $48 \mathrm{~h}$, when the maximum accumulation of this metal was observed. Subsequently, at $72 \mathrm{~h}$, the tissue levels of this metal began to decrease.

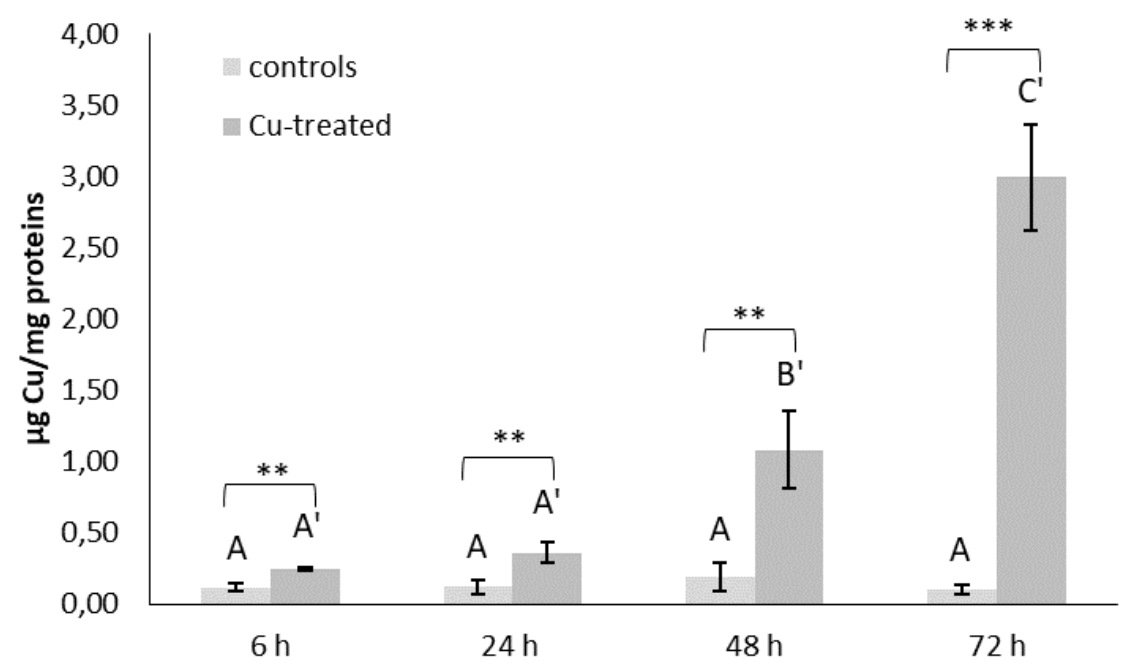

(a) 


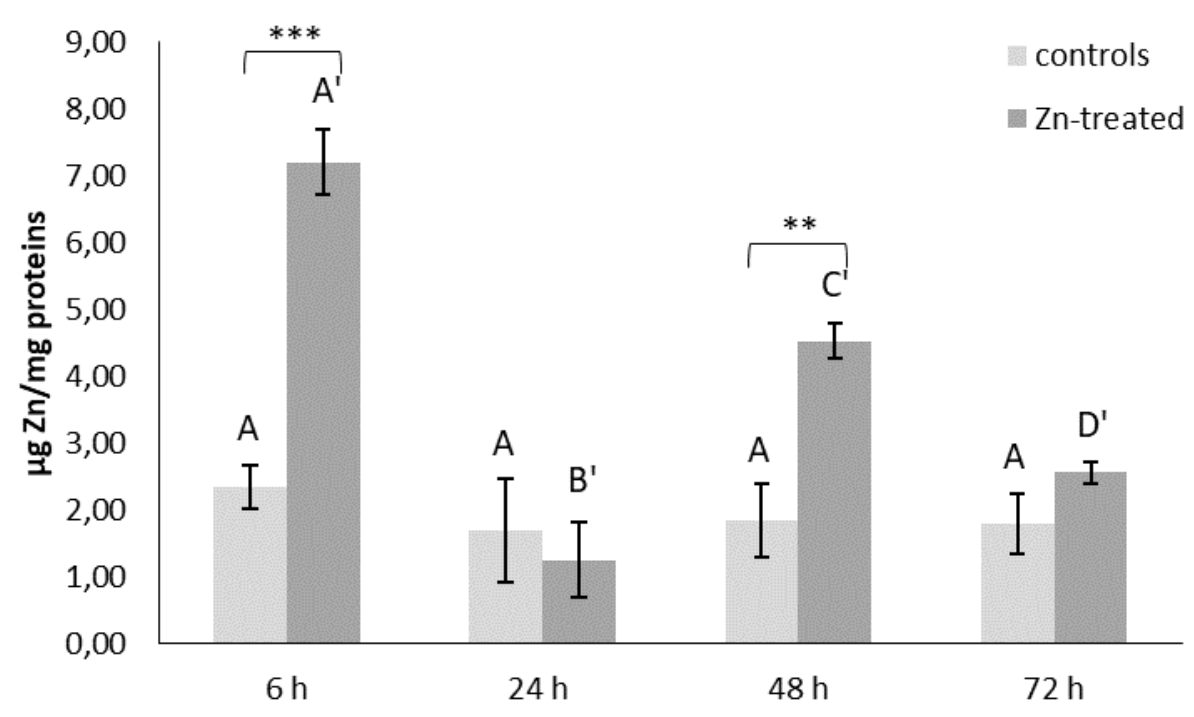

(b)

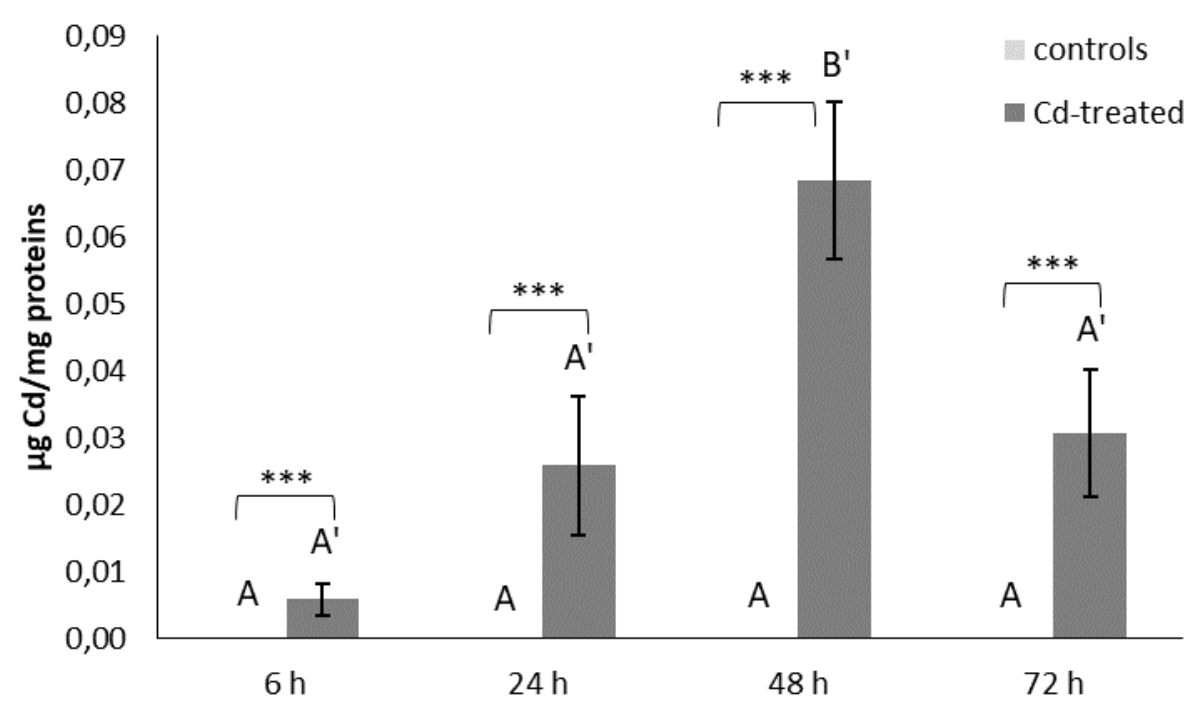

(c)

Figure 6. (a) $\mathrm{Cu},(\mathrm{b}) \mathrm{Zn}$ and (c) $\mathrm{Cd}$ accumulation in the intestine of $\mathrm{C}$. robusta $(\mu \mathrm{g}$ metal/mg total protein). Asterisks: significant differences with respect to controls $\left({ }^{* *} p<0.001,{ }^{* *} p<0.01,{ }^{*} p<\right.$ $0.05)$. Letters with the same index: Student-Newman-Keuls t-comparison among treatments.

\subsection{ISH}

For $c r-P r d x 2$ and $c r-P r d x 3$, the ISH analysis on hemolymph revealed that granular amoebocytes were the only immunocytes expressing the Prdx mRNAs (Figure 7a and b). In the case of $c r-\operatorname{Prdx} 4$, besides granulocytes (Figure 7c), also phagocytes are active in the expression of the transcript (Figure 7d). No labeling was observed with the use of sense riboprobes. 


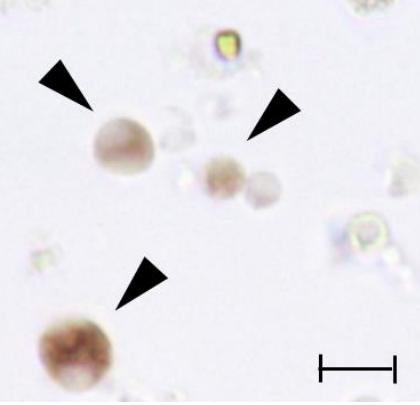

(a)

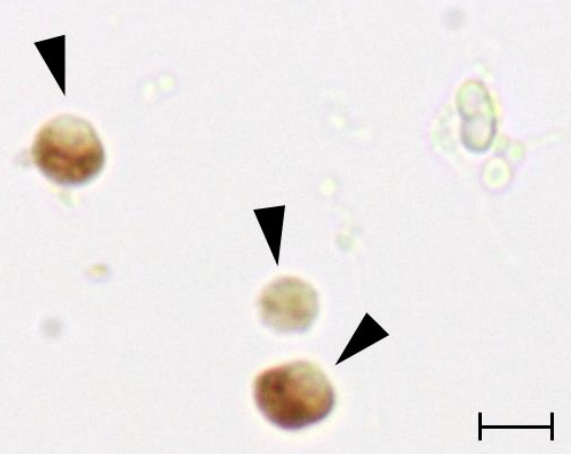

(b)

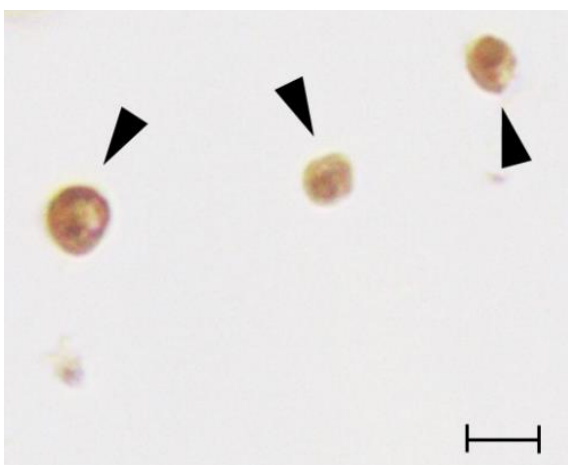

(c)

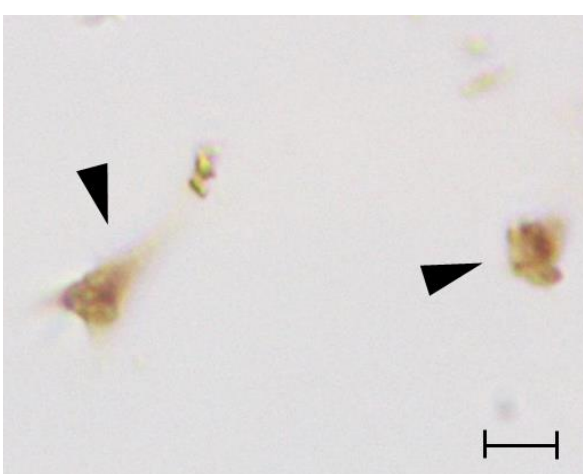

(d) 


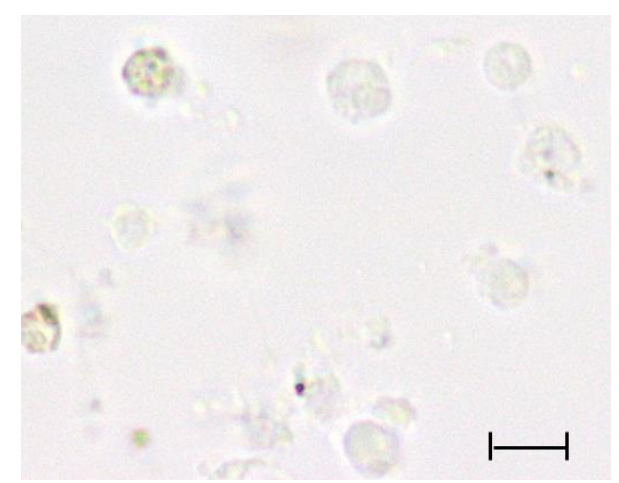

(e)

Figure 7. ISH with anti-sense riboprobes, for (a) $c r-P r d x 2$, (b) $c r-P r d x 3$ and (c, d) $c r-P r d x 4$, and sense riboprobe, for (e) $c r$-Prdx4 as reference, on hemocyte monolayers from three-days Cdtreated. Brown color: riboprobe staining. Arrowheads: labelled hemocytes. Scale bar: $10 \mu \mathrm{m}$.

\section{Discussion}

Marine organisms have adaptations that allow them to maintain homeostasis, ensuring growth and reproduction, in a wide variety of environmental conditions. Like any other metabolic pathway, the processes that lead to the production of ROS also vary significantly, depending on the variations of many environmental factors, and the regulation of antioxidant defenses is essential to keep the concentration of these molecules at sufficiently low levels, in order to prevent oxidative stress and cell death. Many marine animals produce ROS in response to exposure to xenobiotics [47, 48]. The production of ROS and the activation of various components of the antioxidant defense system, such as enzymes that catalyze the biosynthesis of glutathione, superoxide dismutase and glutathione peroxidase, have also been demonstrated in the solitary ascidian Ciona $[35,37,38]$.

Despite a large amount of research on the antioxidant defense system of marine invertebrates, there is still much to discover concerning the molecular components and their mutual functional relationships. The present work characterized the genetic sequences of three Prdxs: $c r-P r d x 2, c r-P r d x 3$, and $c r-P r d x 4$. Furthermore, we demonstrated, for the first time, that they are transcribed and expressed as active proteins in the intestine of $C$. robusta. Upon metal exposure, they increase their transcription, and an increase in the Prdx activity of cell extracts is also observed. Through the analysis of 5' UTR, we identified putative regulatory sequences in $c r-P r d x 3$ and $c r-P r d x 4$, i.e., ARE and MRE, the latter of particular importance in the regulation of mRNA transcription in response to metals. This suggests that $C$. robusta has evolved a defense system against oxidative damages induced by metals.

The phylogenetic analyzes carried out on the nucleotide sequences of the three considered Prdx isoforms made it possible to add new information to the molecular evolution of anti-stress proteins. In particular, they confirmed a high level of nucleotide and amino acid conservation, with cladograms that are highly resolved, while maintaining an excellent homogeneity of sequences distribution within the representative clusters of the various taxa. An interesting result is that for none of the three isoforms it was possible to identify the sequences of the tunicates as sister groups of the vertebrate sequences, unlike what has been evidenced by recent phylogenetic studies [49]. However, this finding could have been partially expected because analyzes carried out on other gene sequences $(\gamma$ glutamyl-cysteine ligase, glutathione synthetase, superoxide dismutase, glutathione peroxidase, TIA-1 related nucleolysin, tristetraproline) showed phylogenetic relationships similar to those obtained here [35,37-39]. In addition, there is the difficult determination of the phylogeny of tunicates and their peculiar intermediate position between vertebrates and invertebrates to be considered. In fact, it is not surprising that the positioning of tunicates as sister groups of vertebrates is the result of the most advanced and recent research works [31] and of years of heated scientific debate [50]. Another interesting result is rep- 
resented by the peculiar position of $B$. leachii $\operatorname{Prdx} 2$, which emerges together with the isoform 4 of this species rather than its orthologous proteins. While maintaining the utmost caution in relation to the absence of other molecular data, we could hypothesize a gene duplication event that occurred relatively recently, following the differentiation between the progenitor of Prdx2 and that of Prdx4.

As regards the phylogenetic analyzes performed on the amino acid sequences, cladograms were obtained which show a practically identical topology to that of the cladograms obtained with the nucleotide sequences, despite a lower degree of resolution. This result is in line with the high degree of conservation of amino acid sequences and could be the result of a strong purifying selection acting on them, an evolutionary feature common to other genes encoding antioxidant enzymes [16, 17, 19, 51, 52]. The action of purifying selection is important for the evolution of gene families, as it guarantees the maintenance of function of the encoded proteins. This is also clear from the multi-alignment analysis, which demonstrated that the amino acids, which are essential for their peroxidase activity, are highly conserved in the Prdx of Ciona, where the catalytic tetrad consisting of proline, threonine, cysteine and arginine plays an important role. A histidine, a serine and an aspartic acid are also important for this enzymatic activity.

The results obtained from the qRT-PCR and enzymatic activity analyzes indicate that the genes encoding all the considered Prdx isoforms are expressed in the intestine of $C$. robusta. Of the three isoforms, the two that were most expressed at baseline are $\mathrm{Cr}-\mathrm{Prdx} 2$ and Cr-Prdx4, 1.5 times more than Cr-Prdx3. The former is mainly expressed in the cytoplasm, and its hyperoxidation is believed to be physiologically relevant in relation to inflammatory and necrosis phenomena $[53,54]$. The second resides as a soluble protein in the lumen of the endoplasmic reticulum (ER), an intracellular structure that plays a fundamental role in many metabolic processes including the biosynthesis of lipids and proteins, and in detoxification processes [55]. It is to known that the correct folding of the tertiary structure of proteins occurs in the ER with the formation of disulfide bridges. One of the most conserved enzymes that performs this function is the Endoplasmic Reticulum Oxidoreductin 1 (ERO1), which during its oxidative activity produces $\mathrm{H}_{2} \mathrm{O}_{2}$ [56]. Considering this, it is not surprising that an isoform of Prdx is specifically expressed within the lumen of the RE, where it can exercise its protective function as a peroxide scavenger. Furthermore, it has been confirmed that $\operatorname{Prdx} 4$ is also secreted [57], as a specific signal is present in its N-terminal portion, and therefore its expression is also associated with inflammatory phenomena [58].

The metal sub-lethal exposures bring a generalized increase in gene expression levels for all the three Cr-Prdxs, in the intestine of C. robusta, as revealed by qRT-PCR analyses. $\mathrm{Cd}$ seems to be the metal that primarily induces a generalized, statistically significant, increase in the transcription levels compared to controls of the Cr-Prdx isoforms except Cr-Prdx3. This increase is also reflected at the post-transcriptional level, as detected in the total enzymatic activity of Prdx 2-Cys. Although we cannot discriminate the contribution of each Cr-Prdxs, we may suppose that this result refers especially to Cr-Prdx2, the mRNA accumulation time-course of which is superimposable to that of enzymatic activity, with the maximum peak at $48 \mathrm{~h}$ and minimum peaks at $24 \mathrm{~h}$ and $72 \mathrm{~h}$, when the amount of active proteins achieves the control levels. These two events may be justified by the overexpression of other components of the antioxidant system, as demonstrated, under the same experimental conditions, for the enzymes catalyzing the GSH biosynthesis [35] and for $\mathrm{Cu} / \mathrm{Zn}$ SOD [37]. We also observed that the accumulation of $\mathrm{Cd}$ in the intestine of $\mathrm{C}$. robusta increases starting from $24 \mathrm{~h}$ up to $48 \mathrm{~h}$, when it reaches its maximum, and then decreases again. The reduction in the intracellular concentration of $\mathrm{Cd}$ may be related to its partial accumulation in the granular amoebocytes of the hemolymph, which then migrate into the tunic, the outer covering of tunicates, which becomes a non-systemic storage site, where these cells undergo cell death by apoptosis [36]. ISH on circulating hemocytes showed that the transcripts for $c r-P r d x 2, c r-P r d x 3$, and $c r-P r d x 4$ are mainly located in granular amoebocytes, as demonstrated in previous works [34-37,39], confirming their role as 
circulating detoxification system [59]. For cr-Prdx4, granulocytes are not the only immunocytes involved in the detoxification processes from metals. Also, phagocytes are involved. The role of phagocytes was demonstrated by Franchi et al. [60] in the colonial ascidian Botryllus schlosseri, which are active in the transcription of stress-related genes like glutathione synthase (GS), superoxide dismutase (SOD), and glutathione peroxidase 5.

Also, $\mathrm{Cu}$ induce an increase in the transcription for all the three Cr-Prdx isoforms, particularly $\mathrm{Cr}-\mathrm{Prdx} 2$, even if in a less marked way than $\mathrm{Cd}$. This was a partly unexpected result considering the redox properties of $\mathrm{Cu}$, which catalyze Haber-Weiss and Fenton reactions leading to hydroxyl radicals $(\bullet \mathrm{OH})$ formation, among the most active ROS [61]. A consistent increase in enzymatic activity in comparison to controls was revealed during the time course. We hypothesize that the mRNA for the three Cr-Prdx isoforms was readily translated into active protein when the intracellular concentration of $\mathrm{Cu}$ increased, also increasing the risk of oxidative stress. The metal accumulation reaches its maximum at 72 $\mathrm{h}$ when we detect the maximum mRNA expression for $\mathrm{Cr}-\mathrm{Prdx} 2$ and $\mathrm{Cr}-\mathrm{Prd} \mathrm{x} 4$. This physiological response seems justified by the fact that the $\mathrm{Cu}$ is an essential metal, so it can only damage the cell when its concentration increases beyond a threshold.

Exposure to $\mathrm{Zn}$ also has much less marked effects on the transcription of genes encoding Cr-Prdx isoforms 2, 3, and 4 than $\mathrm{Cd}$. The maximum increase in mRNA expression levels is observed at $6 \mathrm{~h}$, in concomitance with a peak of the metal accumulation. After that, a general decrease occurs which, for $\mathrm{Cr}-\mathrm{Prdx} 3$, marks the return to control levels. The time-course of intracellular concentrations of $\mathrm{Zn}$ may be a phenomenon similar to that demonstrated for $\mathrm{Cd}$, with partial accumulation in the granular amoebocytes and then extrusion into the tunic [36]. However, the accumulation of active protein begins at $48 \mathrm{~h}$, reaching a maximum peak at $72 \mathrm{~h}$, when the transcription levels for isoforms 2 and 4 seem to increase again. These data suggest a double type of time-dependent regulation on the accumulation of $\mathrm{Zn}$ in C. robusta intestine: in the early exposure, there is a transcriptional regulation, so the transcription for $\mathrm{Cr}-\operatorname{Prdx} 2,3$ and 4 occurs. In the later exposure, there is a post-transcriptional regulation, with the mRNAs translated into active proteins. Our results suggest that part of the transcripts might not immediately be translated, as observed in previous studies on components of the antioxidant system in animals exposed to oxidative stress conditions $[17,51,52,62]$. It is known that there are intracellular compartments, such as P-bodies and stress granules (SGs), in which mRNAs are stored, undergoing degradation or future translation, respectively [63-65]. Our previous study demonstrated the importance of SGs in regulating metal-induced stress responses in $C$. robusta [39]. SG nucleation occurs with the over-expression of mRNA binding proteins, such as TIA-1 related nucleolysin (TIAR) and tristetraprolin (TTP), able to specifically recognize ARE sequences in the promoters for some anti-stress proteins such as Prdxs. The hypothesis is therefore that in the early phase of Zn-exposure, mRNA for Prdxs are stored in SGs, which block their translation. In the later phase, the acute stress is perceived by the cell and induces the disassembly of SGs, since transcription levels of mRNA binding proteins such as TIAR and TTP decrease so to unlock of the translation for Prdxs.

\section{Conclusions}

Our data on the time-course of transcription levels and active proteins levels highlight that $\mathrm{Cr}-\mathrm{Prdx} 2, \mathrm{Cr}-\mathrm{Prdx} 3$, and $\mathrm{Cr}-\mathrm{Prd} \mathrm{x} 4$ perform their antioxidant function in an integrated way. Regarding $\mathrm{Cd}$ and $\mathrm{Cu}, \mathrm{Cr}-\mathrm{Prdx} 2$ is the most active isoform, between the three we studied, in contrasting acute stress induced by the metals. In the presence of $\mathrm{Zn}$, we hypothesize a post-transcriptional control operated by stress granules. Our results, clarifying the importance of peroxiredoxins in protecting organisms from heavy metal pollution, are the first obtained in tunicates. Future studies will characterize and study, at the transcriptional and post-transcriptional level, all the peroxiredoxin isoforms of C. robusta, the putative gene sequences present in the ANISEED database in order to understand better how they work together in contrasting oxidative stress. In addition, a deeper 
insight into the role of Prdxs in C. robusta biology can provide data valid for their use as stress biomarkers in biomonitoring campaigns of the marine ecosystem, using C. robusta as sentinel organism.

Supplementary Materials: The following are available online at www.mdpi.com/xxx/s1, Figure S1: Cr-Prdx2 cDNA sequence and relative amino acid sequence; $5^{\prime}$ - and $3^{\prime}$-UTR regions are underlined; in grey sequence obtained with the amplicon sequencing; degradation signals are boxed in bold, Figure S2. Cr-Prdx3 cDNA sequence and relative amino acid sequence; 5'- and 3'-UTR regions are underlined; in grey sequence obtained with the amplicon sequencing; polyadenylation signals are boxed in double line; degradation signals are boxed in bold, $\mathrm{Cr}$-Prdx4 cDNA sequence and relative amino acid sequence; 5'- and 3'-UTR regions are underlined; in grey sequence obtained with the amplicon sequencing; polyadenylation signals are boxed in double line; degradation signals are boxed in bold, Figure S4. Schematic Prdx2 domain organization. The AhpC-TSA domain is highlighted in light grey, the 1-cysPrx $\mathrm{C}$ domain is highlighted in dark grey. For C. robusta, alpha helixes and beta sheets are boxed with dotted and continues lines respectively; amino acids of the catalytic center are indicated in bold and underlined. ${ }^{*}=$ identical amino acids (completely preserved), $:=$ very similar amino acids (semi-conservative substitution), .= similar amino acids (conservative substitution); numerals refer to character counts, Figure S5. Schematic Prdx3 domain organization. The AhpC-TSA domain is highlighted in light grey, the 1-cysPrx_C domain is highlighted in dark grey. For C. robusta, alpha helixes and beta sheets are boxed with dotted and continues lines respectively; amino acids of the catalytic center are indicated in bold and underlined. ${ }^{*}=$ identical amino acids (completely preserved), := very similar amino acids (semi-conservative substitution), .= similar amino acids (conservative substitution); numerals refer to character counts, Figure S6. Schematic $\operatorname{Prdx} 4$ domain organization. The AhpC-TSA domain is highlighted in light grey, the 1-cysPrx_C domain is highlighted in dark grey. For C. robusta, alpha helixes and beta sheets are boxed with dotted and continues lines respectively; amino acids of the catalytic center are indicated in bold and underlined. * identical amino acids (completely preserved), := very similar amino acids (semi-conservative substitution), .= similar amino acids (conservative substitution); numerals refer to character counts, Figure S7. Phylogenetic relationships among Prdx isoforms of various organisms reconstructed on the amino acid sequences and using both Bayesian interference (BI) and Maximum likelihood (ML) methods. Bayesian posterior probability (first number) and bootstrap values higher (and equal to) than $50 \%$ are indicated on each node, respectively. The scale for branch length (2.0 substitution/site) is shown below the tree, Table S1: Primer sequences, and relative melting temperatures (Tm), used for reverse transcription (Oligo-dT Anchor primer) and for PCR amplification, Table S2: Primer sequences, and relative melting temperatures (Tm), used for qRT-PCR amplification, Table S3. GenBank accession numbers and ANISEED transcript IDs (Ciona robusta, Ciona intestinalis and Botrylloides leachii) referred to amino acid sequences of Prdx2, Prdx3 and Prdx4 used for multi-alignment and phylogenetic analyses, Table S4. Percentages of identity, similarity and E-values obtained by comparing Cr-Prdx2 amino acid sequence with orthologous sequences of metazoans, Table S5. Percentages of identity, similarity and E-values obtained by comparing Cr-Prdx3 amino acid sequence with orthologous sequences of metazoans.

Author Contributions: Conceptualization, Gianfranco Santovito and Loraino Ballarin; methodology, Gianfranco Santovito, Loraino Ballarin and Rigers; validation, Laura Drago; formal analysis, Gianfranco Santovito, Loriasno Balarin, Rigers Bakiu, Diana Ferro, Laura Drago; investigation, Gianfranco Santovito, Loriano Ballarin, Rigers Bakiu, Diana Ferro and Laura Drago; resources, Gianfranco Santovito, Loriano Ballarin and Rigers Bakiu; data curation, Diana Ferro; writing - original draft preparation, Gianfranco Santovito, Loriano Ballarin, Diana Ferro, Laura Drago and Rigers Bakiu; writing - review and editing, Gianfranco Santovito and Loriano Ballarin; visualization, Gianfranco Santovito and Loriano Ballarin; supervision, Gianfranco Santovito and Loriano Ballarin; project administration, Gianfranco Santovito; funding acquisition, Gianfranco Santovito and Loriano Ballarin. All authors have read and agreed to the published version of the manuscript.

Funding: The work was supported by a grant from the Italian MIUR (DOR2018) to G.S. and L.B.

Conflicts of Interest: The authors declare no conflict of interest. 


\section{References}

1. Lesser, M.P. Oxidative stress in marine environments: Biochemistry and physiological ecology. Annu. Rev. Physiol. 2006, 68, 253278. doi:10.1146/annurev.physiol.68.040104.110001.

2. Boldrin, F.; Santovito, G.; Formigari, A.; Bisharyan, Y.; Cassidy-Hanley, D.; Clark, T.G.; Piccinni, E. MTT2, a copper-inducible metallothionein gene from Tetrahymena thermophila. Comp. Biochem. Physiol. C. 2008, 147, 232-240. doi:10.1016/j.cbpc.2007.10.002.

3. Santovito, G.; Piccinni, E.; Irato, P. An improved method for rapid determination of the reduced and oxidized states of metallothioneins in biological samples. In Marine Pollution: New Research; Tobias N. Hofer, Ed; Nova Science Publishers Inc.: New York, USA, 2008; Ch. 3, pp. 101-123.

4. Santovito, G.; Piccinni, E.; Boldrin, F.; Irato, P. Comparative study on metal homeostasis and detoxification in two Antarctic teleosts. Comp. Biochem. Physiol. C. 2012, 155, 580-586. doi:10.1016/j.cbpc.2012.01.008.

5. Santovito, G.; Boldrin, F.; Irato, P. Metal and metallothionein distribution in different tissues of the Mediterranean clam Venerupis philippinarum during copper treatment and detoxification. Comp. Biochem. Physiol. C. 2015, 174-175, 46-53. doi:10.1016/j.cbpc.2015.06.008.

6. Ferro, K.; Ferro, D.; Corrà, F.; Bakiu, R.; Santovito, G.; Kurtz, J. Cu,Zn SOD genes in Tribolium castaneum: evolution, molecular characterisation and gene expression during immune priming. Front. Immunol. 2017, 8, 1811. doi:10.3389/fimmu.2017.01811.

7. Ricci, F.; Lauro, F.M.; Grzymski, J.J.; Read, R.; Bakiu, R.; Santovito, G.; Luporini, P.; Vallesi, A. The anti-oxidant defense system of the marine polar ciliate Euplotes nobilii: characterization of the msrB gene family. Biology. 2017, 6, 4. doi:10.3390/biology6010004.

8. Garofalo, F.; Santovito, G.; Amelio, D. Morpho-functional effects of heat stress on the gills of Antarctic T. bernacchii and C. hamatus. Mar. Poll. Bull. 2019, 141, 194-204. doi: 10.1016/j.marpolbul.2019.02.048.

9. Bukola, D.; Zaid, A. Consequences of anthropogenic activities on fish and the aquatic environment. Poult. Fish. Wildl. Sci. 2015, 3, 138. doi:10.4172/2375-446X.1000138.

10. Dey, S.; Sidor, A.; O'Rourke, B. compartment-specific control of reactive oxygen species scavenging by antioxidant pathway enzymes. J. Biol. Chem. 2016, 291, 11185-97. doi:10.1074/jbc.M116.726968.

11. Abbas, M.N.; Kausar, S.; Cui, H. The biological role of peroxiredoxins in innate immune responses of aquatic invertebrates. Fish. Shellfish. Immun. 2019, 89, 91-97. doi:10.1016/j.fsi.2019.03.062.

12. Detienne, G.; de Haes, W.; Mergan, L.; Edwards, S.L.; Temmerman, L.; van Bael, S. Beyond ROS clearance: peroxiredoxins in stress signaling and aging. Ageing. Res. Rev. 2018, 44, 33-48. doi:10.1016/j.arr.2018.03.005.

13. Fujii, J.; Ikeda, Y. Advances in our understanding of peroxiredoxin, a multifunctional, mammalian redox protein. Redox. Rep. 2002, 7, 123-130. doi:10.1179/135100002125000352.

14. Kusakisako, K.; Hernandez, E.P.; Talactac, M.R.; Yoshii, K.; Umemiya-Shirafuji, R.; Fujisaki, K.; Tanaka, T. Peroxiredoxins are important for the regulation of hydrogen peroxide concentrations in ticks and tick cell line. Ticks. Tick-borne. Dis. 2018, 9, 872881. doi:10.1016/j.ttbdis.2018.03.016.

15. Perkins, A.; Nelson, K.J.; Parsonage, D.; Poole, L.B.; Karplus, P.A. Peroxiredoxins: guardians against oxidative stress and modulators of peroxide signaling. Trends. Biochem. Sci. 2015, 40, 435-45. doi:10.1016/j.tibs.2015.05.001.

16. Bakiu, R.; Santovito, G. New insights into the molecular evolution of metazoan peroxiredoxins. Acta Zool. Bulg. 2015, 67, 305317.

17. Tolomeo, A.M.; Carraro, A.; Bakiu, R.; Toppo, S.; Place, S.P.; Ferro, D.; Santovito, G. Peroxiredoxin 6 from the Antarctic emerald rockcod: molecular characterization of its response to warming. J. Comp. Physiol. 2016, 186B, 59-71. doi:10.1007/s00360-015-09353.

18. Al-Asadi, S.; Malik, A.; Bakiu, R.; Santovito, G.; Schuller, K. Characterization of the peroxiredoxin 1 subfamily from Tetrahymena thermophila. Cell. Mol. Life Sci. 2019, 76, 4745-4768. doi:0.1007/s00018-019-03131-3.

19. Tolomeo, A.M.; Carraro, A.; Bakiu, R.; Toppo, S.; Garofalo, F.; Pellegrino, D.; Gerdol, M.; Ferro, D.; Place, S.P.; Santovito, G. Molecular characterization of novel mitochondrial peroxiredoxins from the Antarctic emerald rockcod and their gene expression in response to environmental warming. Comp. Biochem. Physiol. 2019, C255, 108580. doi:10.1016/j.cbpc.2019.108580.

20. Knoops, B.; Loumaye, E.; Van Der Eecken, V. Knoops B., Loumaye E., Van Der Eecken V. Evolution of the peroxiredoxins. In Peroxiredoxin Systems; Flohé L., Harris J.R., Eds; Subcellular Biochemistry: Springer, Dordrecht, 2007; Vol. 44. doi:10.1007/978-14020-6051-9_2.

21. Henderson, D.; Huebner, C.; Markowitz, M.; Taube, N.; Harvanek, Z.M.; Jakob, U.; Knoefler, D. Do developmental temperatures affect redox level and lifespan in C. elegans through upregulation of peroxiredoxin? Redox. Biol. 2018, 14, 386-390. doi:10.1016/j.redox.2017.10.003.

22. Rhee, S.G.; Woo, H.A.; Kil, I.S.; Bae, S.H. Peroxiredoxin functions as a peroxidase and a regulator and sensor of local peroxides. J. Biol. Chem. 2012, 287, 4403-10. doi:10.1074/jbc.R111.283432.

23. Poole, L.B.; Nelson, K.J. Distribution and features of the six classes of peroxiredoxins. Mol Cells. 2016, 39, 53-9. doi:10.14348/molcells.2016.2330.

24. Hoyle, N.P.; O'Neill, J.S. Oxidation-reduction cycles of peroxiredoxin proteins and non transcriptional aspects of timekeeping. Biochemistry. 2015, 54. doi:10.1021/bi5008386.

25. Wood, ZA; Schröder, E.; Robin Harris, J.; Poole, L.B. Structure, mechanism and regulation of peroxiredoxins. Trends. Biochem. Sci. 2003, 28, 32-40. doi:10.1016/S0968-0004(02)00003-8. 
26. Fisher, A.B. Peroxiredoxin 6: A bifunctional enzyme with glutathione peroxidase and phospholipase a 2 activities. Antioxid. Redox. Sign. 2011, 15, 838-44. doi:10.1089/ars.2010.3412.

27. Hall, A.; Karplus, P.A.; Poole, L.B. Typical 2-Cys Peroxiredoxins - structures, mechanisms and functions. Febs. J. 2009, 276, 246977. doi:10.1111/j.1742-4658.2009.06985.x.

28. Costa, P.M.; Chicano-Gálvez, E.; Caeiro, S.; Lobo, J.; Martins, M.; Ferreira, A.M.; Caetano, M.; Vale, C.; Alhama-Carmona, J.; Lopez-Barea, J. Hepatic proteome changes in Solea senegalensis exposed to contaminated estuarine sediments: a laboratory and in situ survey. Ecotoxicology. 2012, 21, 1194-207. doi:10.1007/s10646-012-0874-7.

29. Xia, X.; Yu, R.; Li, M.; Liu, L.; Zhang, K.; Wang, Y.; Li, B.; Zhang, L.; Song, G.; Zheng, X. Molecular cloning and characterization of two genes encoding peroxiredoxins from freshwater bivalve Anodonta woodiana: antioxidative effect and immune defense. Fish. Shellfish. Immun. 2018, 82, 476-91. doi:10.1016/j.fsi.2018.08.050.

30. Dai, L.S.; Yu, X.M.; Abbas, M.N.; Li, C.S.; Chu, S.H.; Kausar, S.; Wang, T.T. Essential role of the peroxiredoxin 4 in Procambarus clarkii antioxidant defense and immune responses. Fish. Shellfish. Immun. 2018, 75, 216-22. doi:10.1016/j.fsi.2018.01.042.

31. Delsuc, F.; Brinkmann, H.; Chourrout, D.; Philippe, H. Tunicates and not cephalochordates are the closest living relatives of vertebrates. Nature, 2006, 439, 965-8. doi:10.1038/nature04336.

32. Gallo, A.; Tosti, E. The ascidian Ciona intestinalis as model organism for ecotoxicological bioassays. J. Marine. Sci. Res. Dev. 2015, 5, 3. doi:10.4172/2155-9910.1000e138.

33. Dardaillon, J.; Dauga, D.; Simion, P.; Faure, E.; Onuma, T.A.; DeBiasse, M.B.; Louis, A.; Nitta, K.R.; Naville, M.; Besnardeau, L. ANISEED 2019: 4D exploration of genetic data for an extended range of tunicates. Nucleic. Acids. Res. 2019, 48, D668-D675. doi:10.1093/nar/gkz955.

34. Franchi, N.; Boldrin, F.; Ballarin, L.; Piccinni, E. CiMT-1, an unusual chordate metallothionein gene in Ciona intestinalis genome: Structure and expression studies. J. Exp. Zool. Part. A. 2011, 315A, 90-100. doi:10.1002/jez.653.

35. Franchi, N.; Ferro, D.; Ballarin, L.; Santovito, G. Transcription of genes involved in glutathione biosynthesis in the solitary tunicate Ciona intestinalis exposed to metals. Aquat. Toxicol. 2012, 114-115, 14-22. doi:10.1016/j.aquatox.2012.02.007.

36. Franchi, N.; Piccinni, E.; Ferro, D.; Basso, G.; Spolaore, B.; Santovito, G.; Ballarin, L. Characterization and transcription studies of a phytochelatin synthase gene from the solitary tunicate Ciona intestinalis exposed to cadmium. Aquat. Toxicol. 2014, 152, 4756. doi:10.1016/j.aquatox.2014.03.019.

37. Ferro, D.; Franchi, N.; Mangano, V.; Bakiu, R.; Cammarata, M.; Parrinello, N.; Santovito, G.; Ballarin, L. Characterization and metal-induced gene transcription of two new copper zinc superoxide dismutases in the solitary ascidian Ciona intestinalis. Aquat. Toxicol. 2013, 140-141, 369-79. doi:10.1016/j.aquatox.2013.06.020.

38. Ferro, D.; Franchi, N.; Bakiu, R.; Ballarin, L.; Santovito, G. Molecular characterization and metal induced gene expression of the novel glutathione peroxidase 7 from the chordate invertebrate Ciona robusta. Comp. Biochem. Phys. C. 2018, $205,1-7$. doi:10.1016/j.cbpc.2017.12.002.

39. Drago, L.; Peronato, A.; Franchi, N.; Ballarin, L.; Bakiu, R.; Santovito, G. Stress granules in Ciona robusta: first evidences of tia-1related nucleolysin and tristetraprolin gene expression under metal exposure. Comp. Biochem. Phys. C. 2021, $243,108977$. doi:10.1016/j.cbpc.2021.108977.

40. Pfaffl, M.W. A new mathematical model for relative quantification in real-time RT-PCR. Nucleic. Acids. Res. 2001, 29 , e45. doi:10.1093/nar/29.9.e45.

41. Lowry, O.H.; Rosebrough, N.J.; Farr, A.L.; Randall, R.J. Protein measurement with the folin phenol reagent. J. Biol. Chem. 1951, 193, 265-275. doi:10.1016/S0021-9258(19)52451-6.

42. Notredame, C.; Higgins, D.G.; Heringa, J. T-Coffee: a novel method for fast and accurate multiple sequence alignment. J. Mol. Biol. 2000, 302, 205-17. doi:10.1006/jmbi.2000.4042.

43. Darriba, D.; Taboada, G.L.; Doallo, R.; Posada, D. JModelTest 2: more models, new heuristics and parallel computing. Nat. Methods. 2012, 9, 772. doi:10.1038/nmeth.2109.

44. Darriba, D.; Taboada, G.L.; Doallo, R.; Posada, D. ProtTest 3: fast selection of best-fit models of protein evolution. Bioinformatics. 2011, 27, 1164-1165. doi:10.1093/bioinformatics/btr088.

45. Ronquist, F.; Teslenko, M.; van der Mark, P.; Ayres, D.L.; Darling, A.; Höhna, S.; Larget, B.; Liu, L.; Suchard, M.A.; Huelsenbeck, J.P. MrBayes 3.2: efficient bayesian phylogenetic inference and model choice across a large model space. Syst. Biol. 2012, 61, 53942. doi:10.1093/sysbio/sys029.

46. Guindon, S.; Dufayard, J.F.; Lefort, V.; Anisimova, M.; Hordijk, W.; Gascuel, O. New algorithms and methods to estimate maximum-likelihood phylogenies: assessing the performance of PhyML 3.0. Syst. Biol. 2010, 59, 307-21. doi:10.1093/sysbio/syq010.

47. Winston, G. W.; Livingstone, D. R.; Lips, F. Oxygen reduction metabolism by the digestive gland of the common marine mussel, Mytilus edulis. J. Exp. Zool. 1990, 255, 296-308.

48. Bacanskas, L.R.; Whitaker, J.; Di Giulio, R.T. Oxidative stress in two populations of killifish (Fundulus heteroclitus) with differing contaminant exposure histories. Mar. Env. Res. 2004, 58, 597-601. doi:10.1016/j.marenvres.2004.03.048.

49. Stach, T.; Braband, A.; Podsiadlowski, L. Erosion of phylogenetic signal in tunicate mitochondrial genomes on different levels of analysis. Mol. Phylogenet. Evol. 2010, 55, 860-870. doi:10.1016/j.ympev.2010.03.011.

50. Turbeville, J.M.C.; Schulz, J.R.; Raff, R.A. Deuterostome phylogeny and the sister group of the chordates: Evidence from molecules and morphology. Mol. Biol. Evol. 1994, 11, 648-655. doi:10.1093/oxfordjournals.molbev.a040143.

51. Chatzidimitriou, E.; Bisaccia, P.; Corrà, F.; Bonato, M.; Irato, P.; Manuto, L.; Toppo, S.; Bakiu, R.; Santovito, G. Copper/zinc superoxide dismutase from the crocodile icefish Chionodraco hamatus: Antioxidant defense at constant sub-zero temperature. Antioxidants. 2020, 9, 325. doi:10.3390/antiox9040325. 
52. Ferro, D.; Bakiu, R.; Pucciarelli, S.; Miceli, C.; Vallesi, A.; Irato, P.; Santovito, G. Molecular characterization, protein-protein interaction network, and evolution of four glutathione peroxidases from Tetrahymena thermophila. Antioxidants. $2020,9,949$. doi:10.3390/antiox9100949.

53. Rabilloud, T.; Heller, M.; Gasnier, F.; Luche, S.; Rey, C.; Aebersold, R.; Benahmed, M.; Louisot, P.; Lunardi, J. Proteomics analysis of cellular response to oxidative stress: evidence for in vivo over-oxidation of peroxiredoxins at their active site. J. Biol. Chem. 2002, 277, 19396-401. doi:10.1074/jbc.M106585200.

54. Valero, Y.; Martínez-Morcillo, F.J.; Esteban M.À.; Chaves-Pozo, E.; Cuesta, A. Fish peroxiredoxins and their role in immunity. Biology. 2015, 4, 860-880. doi:10.3390/biology4040860.

55. Zito, E.; Melo, E.P.; Yang, Y.; Wahlander, A.; Neubert, T.A.; Ron, D. Oxidative protein folding by an endoplasmic reticulumlocalized peroxiredoxin. Mol. Cell. 2010, 40, 787-797. doi:10.1016/j.molcel.2010.11.010.

56. Zito E. ERO1: A protein disulfide oxidase and $\mathrm{H}_{2} \mathrm{O}_{2}$ producer. Free. Radical. Bio. Med. 2015, 83, 299-304. doi:10.1016/j.freeradbiomed.2015.01.011.

57. Tavender, T.J.; Sheppard, A.M.; Bulleid, N.J. Peroxiredoxin IV is an endoplasmic reticulum-localized enzyme forming oligomeric complexes in human cells. Biochem. J. 2008, 411, 191-199. doi:10.1242/jcs.067843.

58. Radyuk, S.N.; Klichko, V.I.; Michalak, K.; Orr, W.C. The effect of peroxiredoxin 4 on fly physiology is a complex interplay of antioxidant and signaling functions. Faseb. J. 2013, 27, 1426-1438. doi:10.1096/fj.12-214106.

59. Kobayashi, K.; Shida, R.; Hasegawa, T.; Satoh, M.; Seko, Y.; Tohyama, C.; Kuroda, J.; Shibata, N.; Imura, N.; Himeno, S. Induction of hepatic metallothionein by trivalent cerium: Role of interleukin 6. Biol. Pharm. Bull. 2005, 28, 1859-63. doi:10.1248/bpb.28.1859.

60. Franchi, N.; Ballarin, L. Immunity in protochordates: the tunicate perspective. Front. Immunol. 2017,8 , 674. doi:10.3389/fimmu.2017.00674.

61. Halliwell, B.; Gutteridge, J.M.C. Free radicals in biology and medicine. Acta. Crystallogr. D. 2017, 73, 384-5. doi:10.1107/S2059798317004533.

62. Sattin, G.; Bakiu, R.; Tolomeo, A.M.; Carraro, A.; Coppola, D.; Ferro, D.; Patarnello, T.; Santovito, G. Characterization and expression of a new cytoplasmic glutathione peroxidase 1 gene in the antarctic fish Trematomus bernacchii. Hydrobiologia. 2015, 761, 363-72. doi:10.1007/s10750-015-2488-6.

63. Waris, S.; Wilce, M.; Wilce, J. RNA recognition and stress granule formation by TIA proteins. Int. J. Mol. Sci. 2014, 15, $23377-88$. doi:10.3390/ijms151223377.

64. Lavut, A.; Raveh, D. Sequestration of highly expressed mrnas in cytoplasmic granules, p-bodies, and stress granules enhances cell viability. PLoS Genetics. 2012, 8, e1002527. doi:10.1371/journal.pgen.1002527.

65. van Treeck, B.; Parker, R. Principles of stress granules revealed by imaging approaches. CSH. Perspect. Biol. 2019, 11, a033068. doi:10.1101/cshperspect.a033068. 